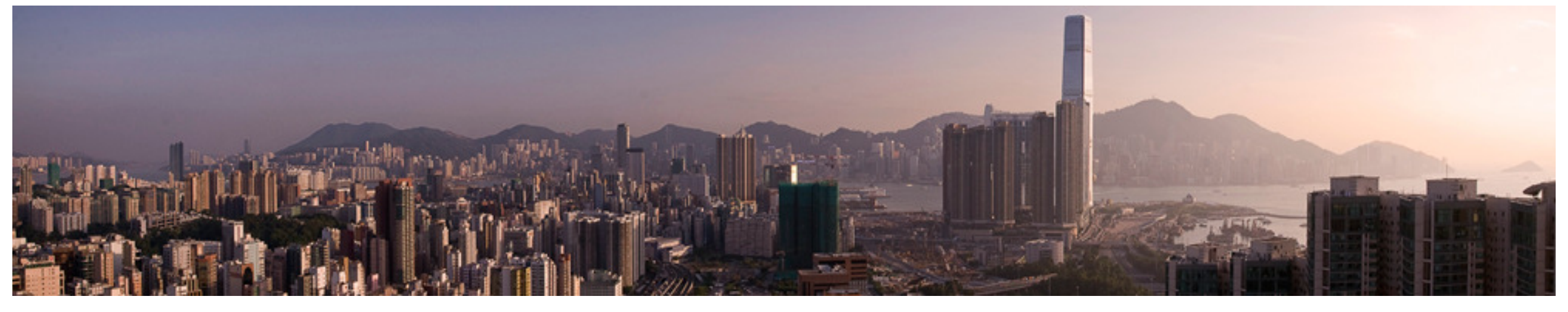

\title{
Recommendation \\ in Social Networks
}

\author{
Martin Ester
}

Simon Fraser University

Tutorial at RecSys 2013 


\section{Outline}

- Introduction

- Recommendation in social networks

- Memory based approaches for item recommendation

- Model based approaches for item recommendation

- Top-N item recommendation

- Friend recommendation

- Future research 


\section{Introduction}

- Users want to have personalized results.

- But are not willing to spend a lot of time to specify their personal information needs.

- Recommender systems automatically identify information relevant for a given user, learning from available data.

- Data

- user actions,

- user profiles. 


\section{Introduction}

\section{Rating Matrix}

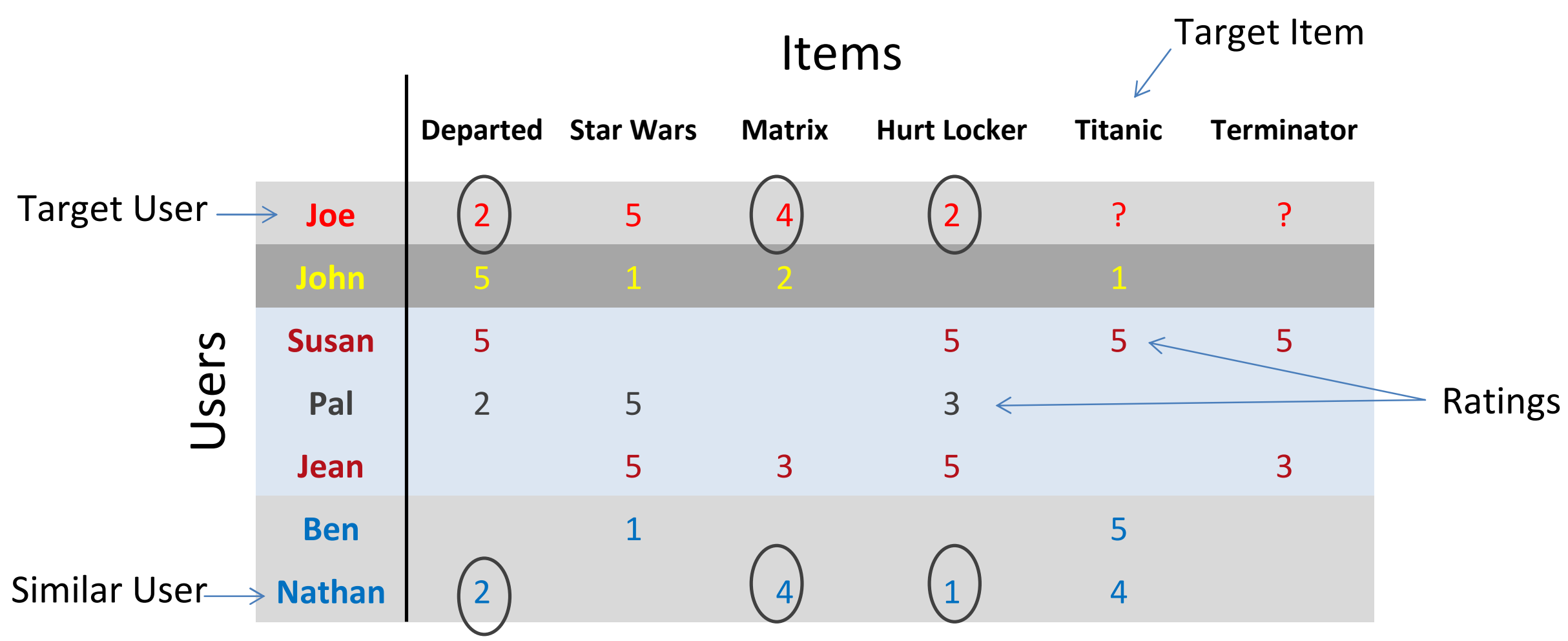




\section{Introduction}

- Rating prediction

Predict the rating of target user for target item, e.g. predict Joe's rating for Titanic.

- Top- $\mathrm{N}$ item recommendation

Predict the top- $\mathrm{N}$ highest-rated items among the items not yet rated by target user.

- Friend recommendation (only if social network) Predict the top-N users to which the target user is most likely to connect. 


\section{Social Networks}

[Wasserman \& Faust 1994]

- Used widely in the social and behavioral sciences, in economics, marketing, ... .

- Directed or undirected graph nodes: actors edges: social relationships or interactions

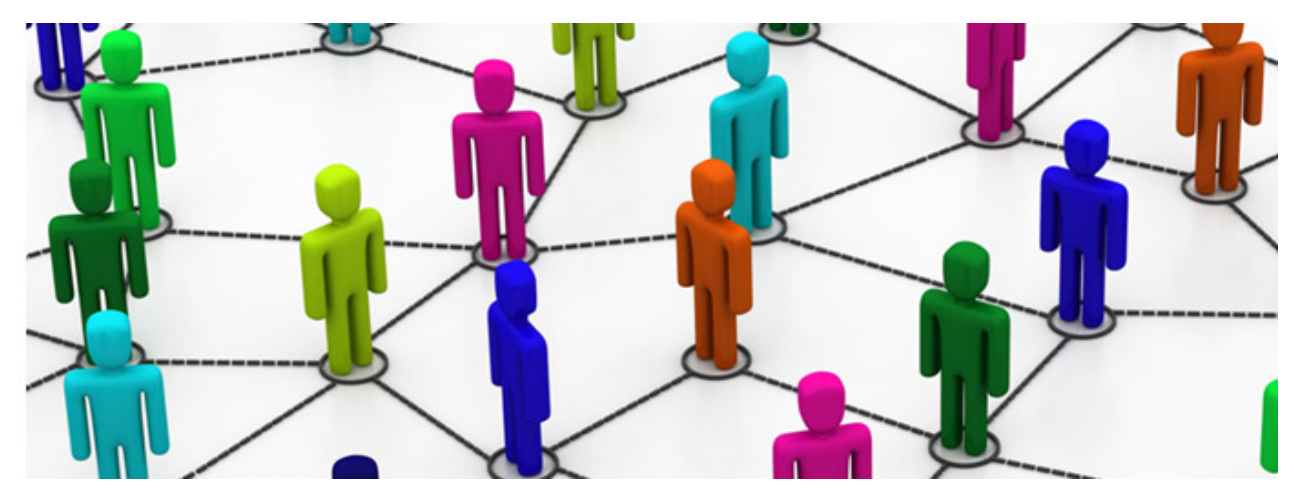




\section{Social Networks}

- Different types of social relationships

- Different types of interactions

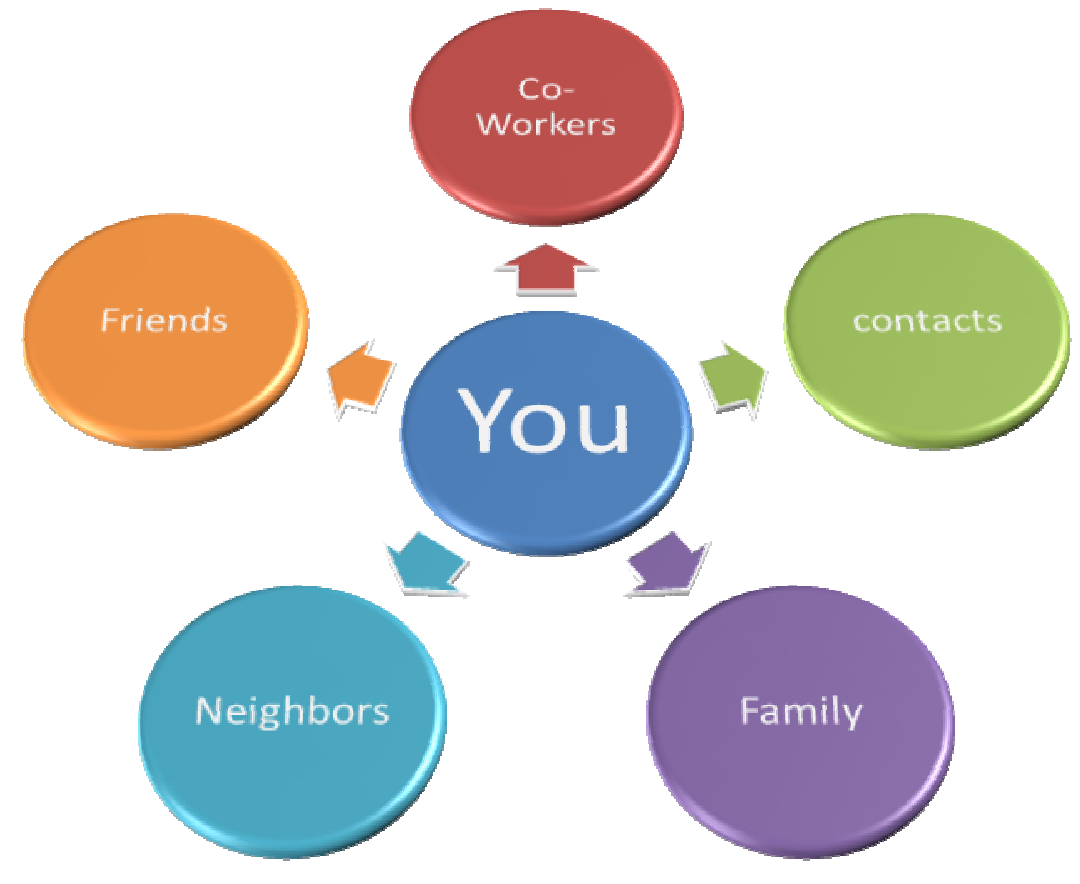




\section{Social Networks}

- Explicit social network relationships provided by users

\section{facebook}

Linkedin.

- Implicit social network

relationships inferred from user actions

- Email network

- Co-worker network 


\section{Social Networks}

[Monge \& Contractor 2003]

- The formation and evolution of social networks is affected by many effects, including

- Self-interest,

- Social and resource exchange,

- Balance,

- Homophily,

-Proximity. 


\section{Trust Networks}

[Golbeck 2005]

- Trust network allows users to

- systematically document their trust-relationships,

- see which users have declared trust in another user.

- Connected users do not necessarily have a social relationship.

- Trust in a user may be based, e.g., on articles or reviews authored by that user.

- Trusted users influence other users. 


\section{Online Social Networks}

- Emergence of online social networks

- Among the top websites http://www.alexa.com/topsites

2. FaceBook

\section{facebook.}

8. Linkedln

Linked in.

10. Twitter

Ewitber

$\rightarrow$ Availability of very large datasets 


\section{Social Rating Networks}

- Social rating network (SRN):

social network, where users are associated with item ratings.

- Item ratings can be numeric [1..5] or Boolean (bookmark photo, like article, ... .).

- Examples: Epinions, Flixster, last.fm, flickr, Digg.

- Social action: create social relationship, rating action: rate an item. 


\section{Social Rating Networks}

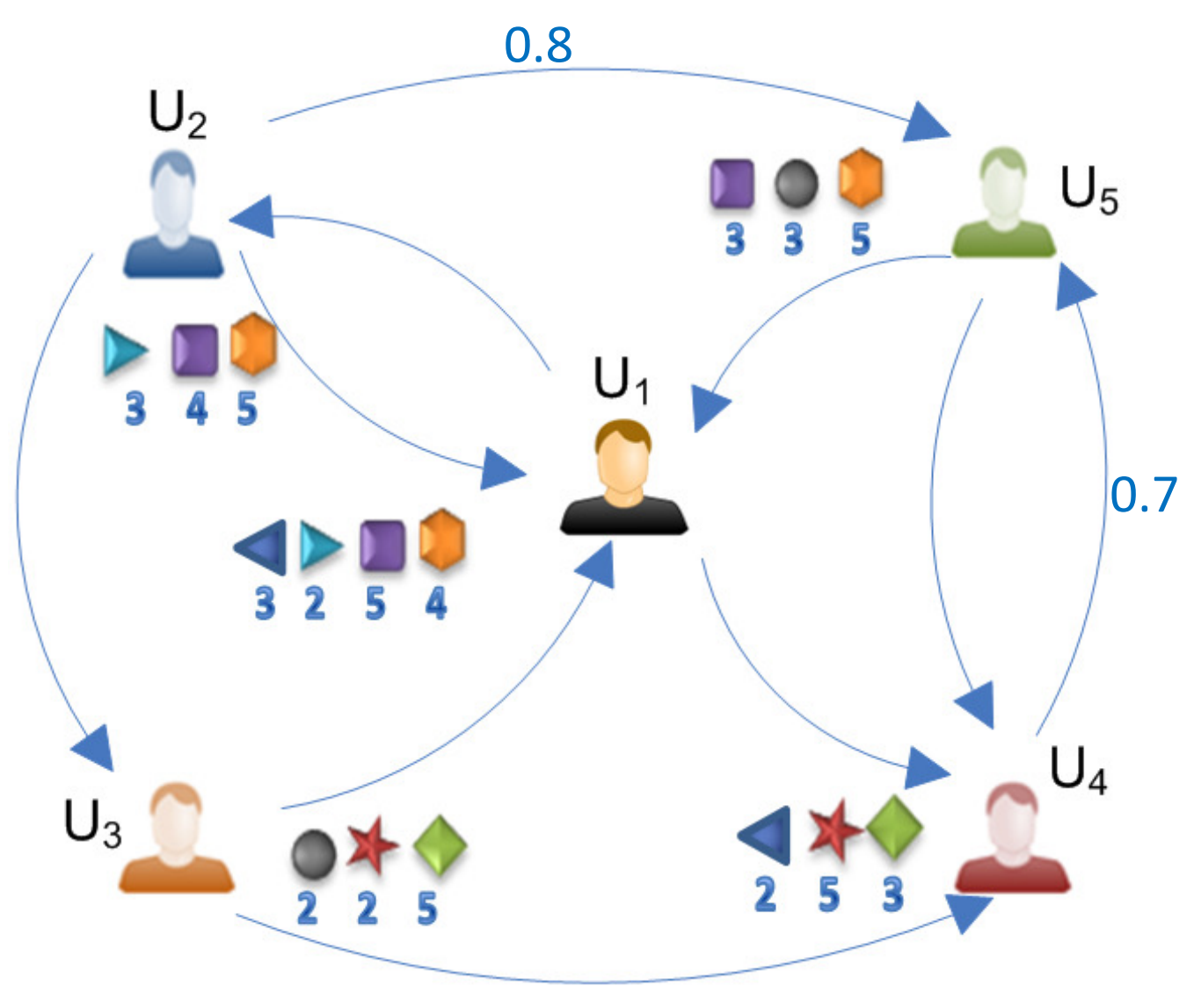




\section{Effects in Social Rating Networks}

SFU

- Social influence:

ratings are influenced by ratings of friends,

i.e. friends are more likely to have similar ratings than strangers.

- Correlational influence: ratings are influenced by ratings of actors with similar ratings,

i.e. if some ratings are similar, further ratings are more likely also to be similar. 


\section{Effects in Social Rating Networks}

SFU

- Selection (homophily):

actors relate to actors with similar ratings,

i.e. actors with similar ratings are more likely

to become friends.

- Transitivity:

actors relate to friends of their friends,

i.e. actors are more likely to relate to indirect friends. 


\section{Recommendation in Social Networks}

- Benefits of social network-based recommendation:

- Exploit social influence, correlational influence, transitivity, selection.

- Can deal with cold-start users, as long as they are connected to the social network.

- Are more robust to fraud, in particular to profile attacks. 


\section{Recommendation in Social Networks}

- Challenges

- Low probability of finding rater of target item at small network distance.

- Ratings at large network distances are noisy.

- Social network data is sensitive (privacy concerns).

-Edges in online social networks are of greatly varying reliability / strength. 


\section{Influence and Correlation}

[Anagnostopoulos et al. 2008]

- Question: does a SN exhibit social influence?

- Discrete time period [0..T], consider only one action, e.g. using a certain tag.

- At every time step, each user flips a coin to decide whether he will get active.

- Probability of activation depends only on number $a$ of already active friends:

$$
p(a)=\frac{e^{\alpha \ln (a+1)+\beta}}{1+e^{\alpha \ln (a+1)+\beta}}
$$




\section{Influence and Correlation}

- $\alpha$ measures social correlation

- $Y_{a, t}:$ number of users with $a$ active friends at time $t-1$ who get activated at time $t$

- $N_{a, t}:$ number of users with $a$ active friends at time $t-1$ who do not get activated at time $t$

- $Y_{a}=\sum_{t} Y_{a, t}, N_{a}=\sum_{t} N_{a, t}$

- Compute $\alpha$ and $\beta$ that maximize the data likelihood $\prod_{a} p(a)^{Y_{a}}\left(1-(p(a))^{N_{a}}\right.$ 


\section{Influence and Correlation}

- If social influence plays a role, then the timing of activation should depend on the timing of activation of other users.

- $W=\left\{w_{1}, \ldots, w_{l}\right\}$ : set of active users at time T

- $t_{i}$ : activation time of user $i$

- Shuffle test

- Perform random permutation $\pi$ of $\{1, \ldots, l\}$.

- Set activation time of user $i$ to $t_{i^{\prime}}:=t_{\pi(i)}$. 


\section{Influence and Correlation}

- Compute $\alpha$ for original activation times.

- Compute $\alpha^{\prime}$ for shuffled activation times.

- If $\alpha$ and $\alpha^{\prime}$ are close to each other, then the model exhibits no social influence.

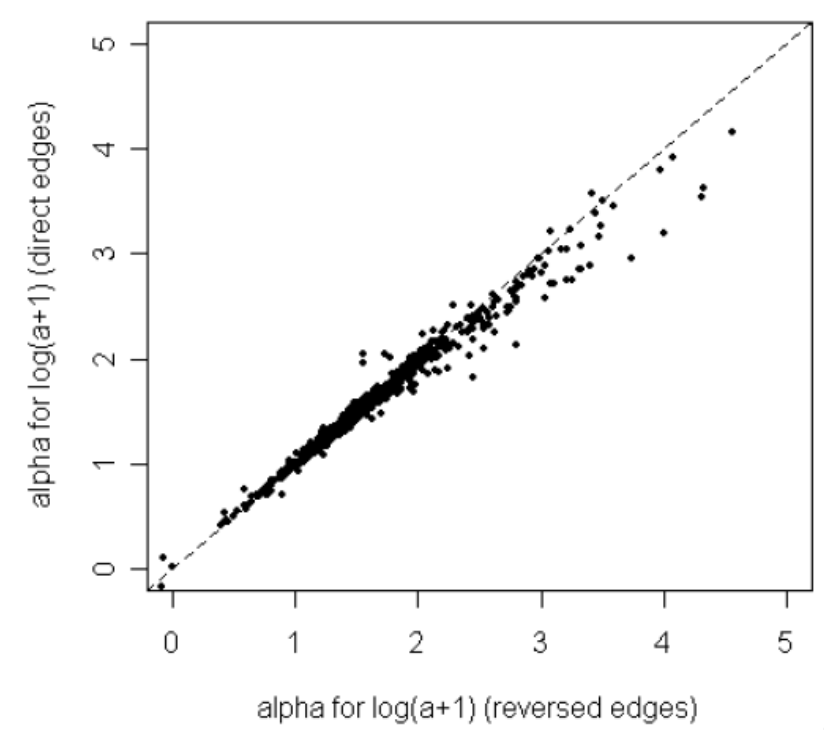

$\alpha$ for original activation times vs. $\alpha^{\prime}$ for shuffled activation times on Flickr dataset

$\rightarrow$ Social correlation, but no social influence! 


\section{Inferring Social Relationships and Their Strength}

- Often: (Boolean) social network given.

- Sometimes, no information about social relationships, only user actions.

$\rightarrow$ Inference of social network from user actions

- Often, users have many friends, and the strength of the relationships varies greatly.

$\rightarrow$ Inference of weighted social network 


\section{Inferring Social Networks}

SFU

[Gomez-Rodriguez et al. 2010]

- Goal: infer social relationships from user actions with time stamps.

- Assumption: there is a latent, static network over which influence propagates.

- $t_{u}$ : activation time of user $u$, i.e. time when user $u$ gets activated ("infected") by a cascade

- Cascade $c$ specified through activation times of all users: $c=\left[t_{1}, \ldots, t_{n}\right]$, possibly $t_{i}=\infty$ 


\section{Inferring Social Networks}

- Independent Cascade model:

activated node activates each of his friends with

a given probability

- $P_{c}(u, v)$ : probability of cascade $c$ spreading from user $u$ to user $v$

- $\Delta=t_{v}-t_{u}$

- $P_{c}(u, v)$ decreases with increasing $\Delta$

$$
P_{c}(u, v) \propto e^{-\frac{\Delta}{\alpha}} \text { or } P_{c}(u, v) \propto \frac{1}{\Delta^{\alpha}}
$$




\section{Inferring Social Networks}

- C: set of all given cascades

- G: inferred directed graph over node (user) set $\mathrm{U}=\{1, \ldots, \mathrm{n}\}$

- $T(G)$ : set of all subtrees of $G$

$$
\begin{aligned}
& P(c \mid T)=\prod_{(u, v) \in T} P_{c}(u, v) \\
& P(C \mid G)=\prod_{c \in C} \max _{T \in T(G)} P(c \mid T)=\prod_{c \in C} \max _{T \in T(G)} \prod_{(u, v) \in T} P_{c}(u, v)
\end{aligned}
$$

- Problem: compute $G$ with at most $k$ edges that maximizes the likelihood $P(C \mid G)$ 


\section{Inferring Social Networks}

- Improvement of log-likelihood over empty graph $E: F_{c}(G)=\max _{T \in T(G)} \log P(c \mid T)-\max _{T \in T(E)} \log P(c \mid T)$

- Equivalent problem

$$
G^{*}=\underset{|G| \leq k}{\operatorname{argmax}} F_{C}(G)
$$

- Problem is NP-hard.

- $F_{C}(G)$ is submodular, which means that a greedy algorithm gives a constant-factor approximation of the optimal solution.

$\rightarrow$ Netlnf algorithm 


\section{Inferring Weighted Social Networks SFU}

[Myers et al. 2010]

- NetInf is very accurate for homogeneous networks, i.e. networks where all connected nodes influence ("infect") each other with the same probability.

- For inhomogeneous networks, define $A_{i j}=P$ (node $i$ infects node $j I$ node $i$ is infected $)$

- Goal: learn the matrix $\mathrm{A}=\left[A_{i j}\right]$ from the observed set of cascades $C=\left\{c_{1}, \ldots, c_{n}\right\}$ 


\section{Inferring Weighted Social Networks}

- If $i$ becomes infected, then $j$ will be infected with probability $A_{i j}$.

- $w(t)$ : transmission time model probability distribution of the transmission time from one node to a friend

$\tau_{i}^{c}$ :time of infection of node $i$ by cascade $c$ time of infection of $i$ 's friend $j$ by cascade $c$

$$
\tau_{j}^{c}=\tau_{i}^{c}+t, \text { where } t \sim w(t)
$$




\section{Inferring Weighted Social Networks}

SFU

- Likelihood of observed cascades $C$ given a weight matrix $A$

$P(C \mid A)=\prod_{c \in C}\left[\left(\prod_{i: \tau_{i}^{c}<\infty}\left(1-\prod_{j: \tau_{j}^{c}<\tau_{i}^{c}}\left(1-w\left(\tau_{i}^{c}-\tau_{j}^{c}\right) A_{j i}\right)\right)\right)\left(\prod_{i: \tau_{i}^{c}=\infty} \prod_{j: \tau_{j}^{c}<\infty}\left(1-A_{j i}\right)\right)\right]$

- First term: one factor for each infected node $i$, assuming that at least one of his friends $j$ who was infected earlier infected him.

- Second term: one factor for each non-infected node $i$, assuming that none of the infected friends $j$ infected whim. : Recommendation in Social Networks, Tutorial at Recsys 2013 


\section{Data Sets for Recommendation in SNS}

SFU

- Epinions

- Online product reviews.

- Explicit notion of trust.

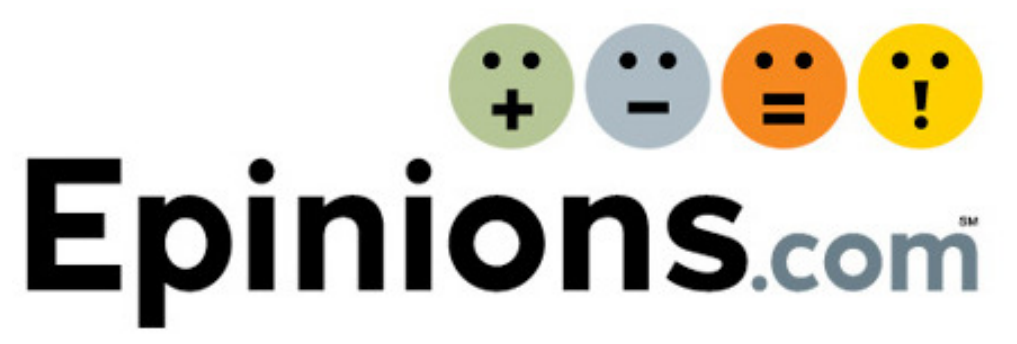

- Users review and rate products in different categories.

- Users express trust on other reviewers.

- http://www.trustlet.org/wiki/Epinions_dataset

- 50K users, $140 \mathrm{~K}$ items, $650 \mathrm{~K}$ ratings, 480K links

- http://alchemy.cs.washington.edu/data/epinions/

- $70 \mathrm{~K}$ users, $105 \mathrm{~K}$ items, 575K ratings, 500K links

$-50 \%$ cold start

- Less than 5 ratings 


\section{Data Sets for Recommendation in SNS $\mathrm{SFU}$}

- Flixster

- Social networking service for rating movies.

- Friendship relations.

- http://www.sfu.ca/ sja25/datasets/

- 1M users, 50K items, 8M ratings, $26 \mathrm{M}$ links

$-85 \%$ of users have no ratings

$-50 \%$ of raters are cold start less than 5 ratings

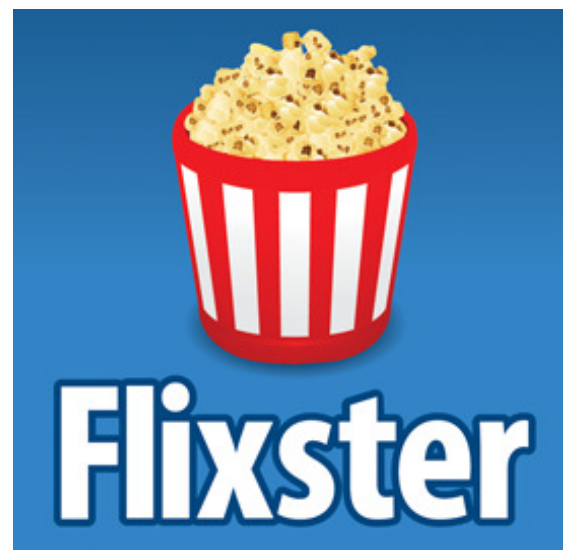




\section{Approaches for Recommendation in SNS}

- Memory based approaches

- Explore the social network for raters.

- Aggregate the ratings to compute prediction.

- Store the social rating network.

- No learning phase.

- Slow in prediction.

- First generation of recommenders in SN were memory based approaches. 


\section{Approaches for Recommendation in $\mathrm{SNS}^{\mathrm{SFU}}$}

- Model based approaches

- Learn a model.

- Store the model parameters only.

- Substantial time for learning.

- Fast in prediction.

- Most methods are based on matrix factorization. 


\section{Memory based Approaches}

- Explore the network to find raters in the neighborhood of the target user.

- Aggregate the ratings of these raters to predict the rating of the target user.

- Different methods to calculate the "trusted neighborhood" of users. 


\section{TidalTrust}

[Golbeck 2005]

- Modified breadth-first search in the network.

- Consider all raters $v$ at the shortest distance from target user $u$.

- Trust between $u$ and $v$

$$
t_{u, v}=\frac{\sum_{w \in N_{u}} t_{u, w} t_{w, v}}{\sum_{w \in N_{u}} t_{u, w}}
$$

where $N_{u}$ denotes set of (direct) neighbors (friends) of $u$

- Trust depends on all connecting paths. 


\section{TidalTrust}

- Predicted rating

$$
\hat{r}_{u, i}=\frac{\sum_{v \in \text { raters }} t_{u, v} r_{v, i}}{\sum_{v \in \text { raters }} t_{u, v}}
$$

where $r_{v, i}$ denotes rating of user $v$ for item $i$

- Only considers raters at the shortest distance:

- Efficient,

- High precision,

- Low recall. 


\section{MoleTrust}

[Massa \& Avesani 2007]

- Similar to the idea of TidalTrust.

- Considers raters up to a maximum-depth $d$.

- Tuning $d$ :

Trade-off between precision (and efficiency) and recall. 


\section{TrustWalker}

[Jamali \& Ester 2009]

- How far to explore the network?

- Instead of far neighbors who have rated the target item use near neighbors who have rated similar items.

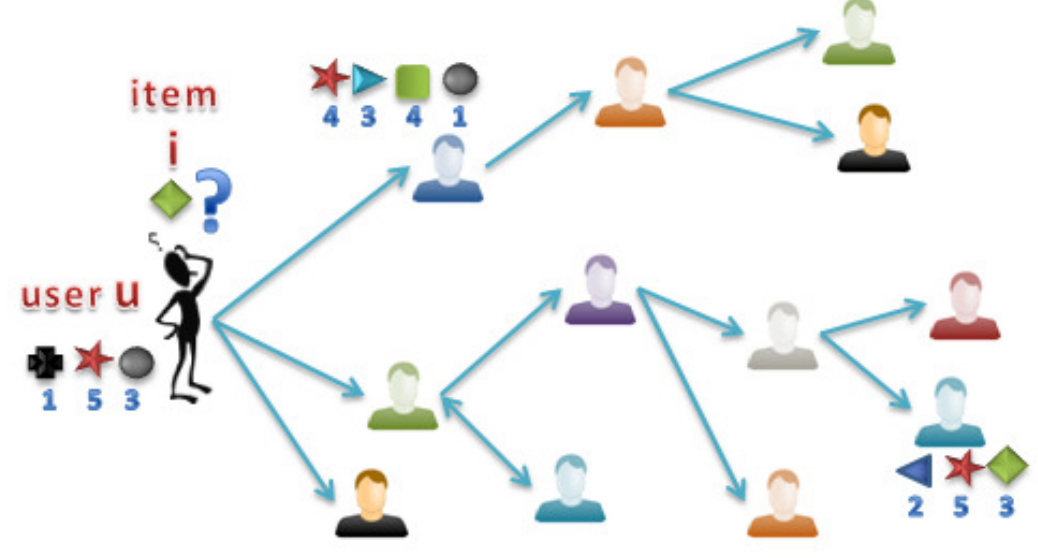




\section{TrustWalker}

- Random walk based model.

- Combines item-based recommendation and trust-based recommendation.

- Performs several random walks on the network.

- Each random walk returns a rating of the target item or a similar item.

- Prediction = aggregate of all returned ratings 


\section{TrustWalker}

- Each random walk starts from target user $u_{0}$.

- At step $k$, at node $u$ :

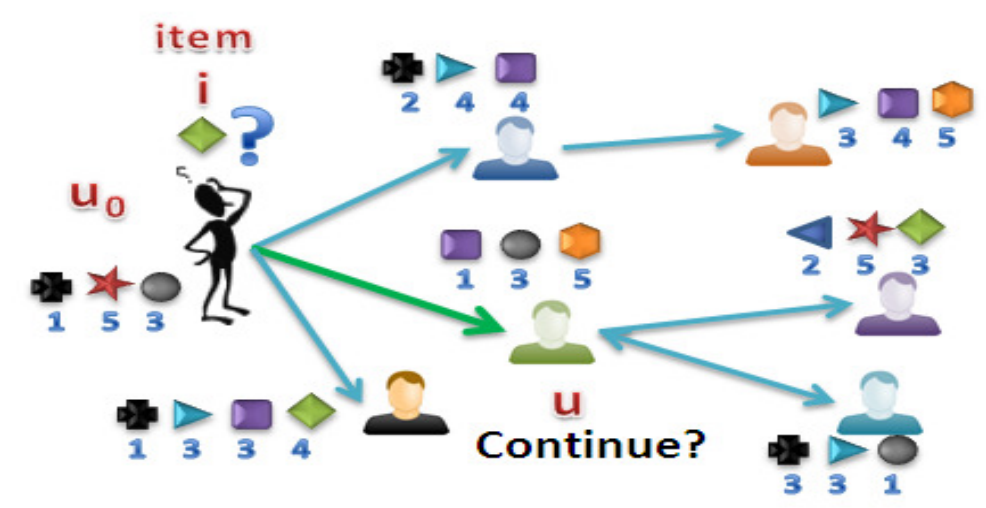

- If $u$ has rated $i$, return $r_{u, l}$.

- With $\Phi_{u, i, k}$, stop random walk, randomly select item $j$ rated by $u$ and return $r_{u, j}$.

- With 1- $\Phi_{u, i, k}$, continue the random walk to a direct neighbor of $u$. 


\section{TrustWalker}

- Item similarities

$$
\operatorname{sim}(i, j)=\frac{1}{1+e^{-\frac{\left|U C_{i, j}\right|}{2}}} \times \operatorname{corr}(i, j)
$$

- $\Phi_{u, i, k}$ depends on

- Similarity of items rated by $u$ and target item $i$

- And the step of random walk:

$$
\phi_{u, i, k}=\max _{j \in R I_{u}} \operatorname{sim}(i, j) \times \frac{1}{1+e^{-\frac{k}{2}}}
$$




\section{Experimental Evaluation}

\section{RMSE results on Epinions}

\begin{tabular}{|c||c|c|c||c|c|c|}
\hline \multirow{2}{*}{\multicolumn{1}{|c||}{ Method }} & \multicolumn{3}{c|}{ Cold Start Users } & \multicolumn{3}{c|}{ All Users } \\
\cline { 2 - 7 } & RMSE & Coverage(\%) & F-Measure & RMSE & Coverage(\%) & F-Measure \\
\hline \hline Item based CF & 1.551 & 21.26 & 0.316 & 1.232 & 68.91 & 0.691 \\
\hline User based CF & 1.498 & 16.34 & 0.259 & 1.277 & 67.54 & 0.688 \\
\hline MoleTrust & 1.441 & 55.36 & 0.594 & 1.104 & 81.03 & 0.765 \\
\hline TidalTrust & 1.223 & 56.92 & 0.626 & 1.109 & 82.37 & 0.770 \\
\hline TrustWalker & 1.201 & 70.17 & 0.701 & 1.079 & 93.22 & 0.819 \\
\hline
\end{tabular}

\section{RMSE results on Flixster}

\begin{tabular}{|c||c|c|c||c|c|c|}
\hline \multirow{2}{*}{ Method } & \multicolumn{3}{c|}{ Cold Start Users } & \multicolumn{3}{c|}{ All Users } \\
\cline { 2 - 7 } & RMSE & Coverage(\%) & F-Measure & RMSE & Coverage(\%) & F-Measure \\
\hline \hline Item based CF & 1.097 & 71.59 & 0.721 & 0.8938 & 94.27 & 0.852 \\
\hline User based CF & 1.114 & 69.86 & 0.710 & 0.9132 & 90.37 & 0.833 \\
\hline MoleTrust & 1.083 & 95.93 & 0.829 & 0.8997 & 95.46 & 0.856 \\
\hline Tidal Trust & 1.106 & 96.11 & 0.826 & 0.8821 & 96.12 & 0.861 \\
\hline TrustWalker & 1.042 & 96.51 & 0.837 & 0.8413 & 99.63 & 0.881 \\
\hline
\end{tabular}




\section{Experimental Evaluation}
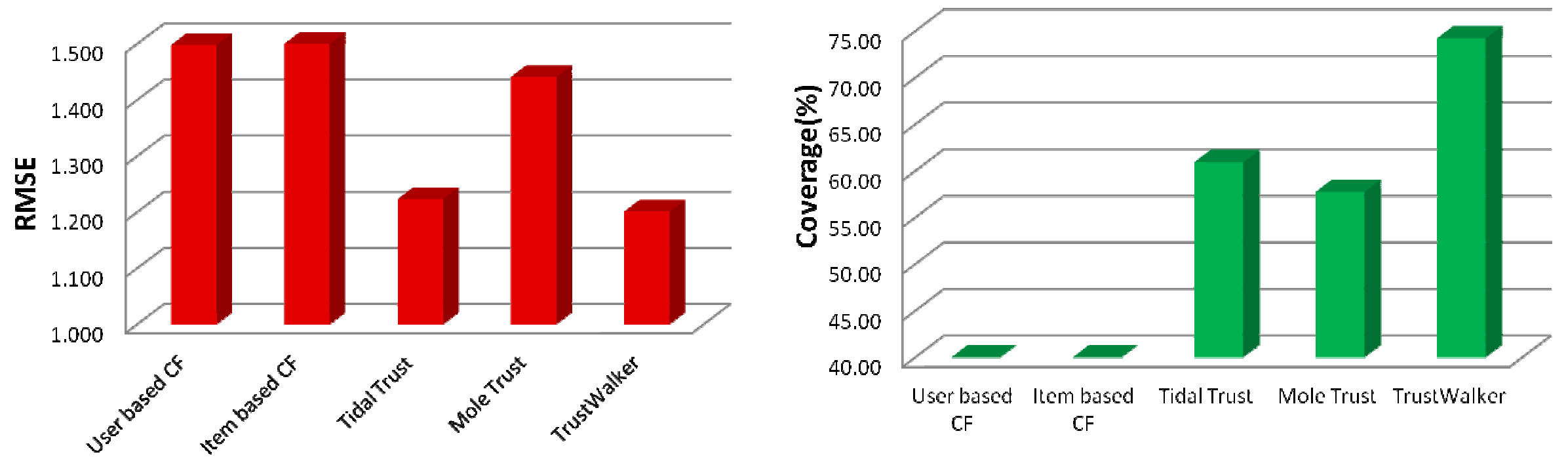

Results for cold start users on Epinions 


\section{Experimental Evaluation}

SFU
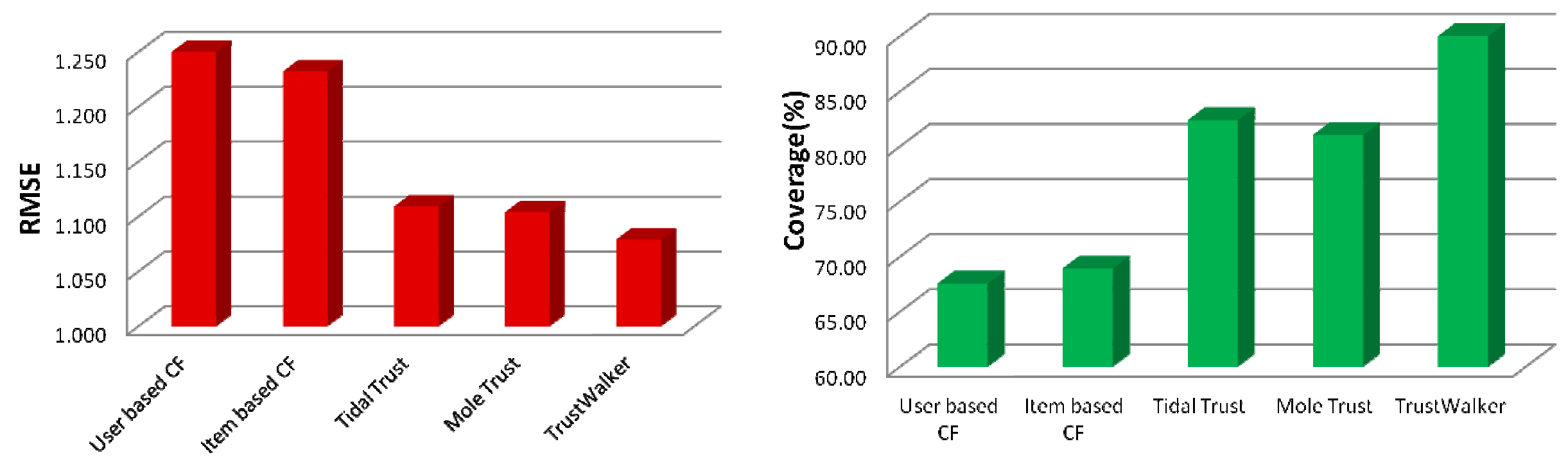

Results for all users on Epinions 


\section{Model based approaches}

- Matrix factorization [Koren et al. 2009]

- Observed ratings $R_{u, i}$

- Latent factors for users

$$
U \in \mathbb{R}^{K \times N}
$$

- Latent factors for items

$$
\begin{gathered}
V \in \mathbb{R}^{K \times M} \\
p\left(R \mid U, V, \sigma_{R}^{2}\right)=\prod_{u=1}^{N} \prod_{i=1}^{M}\left[\mathcal{N}\left(R_{u, i} \mid U_{u}^{T} V_{i}, \sigma_{r}^{2}\right)\right]^{I_{u, i}^{R}}
\end{gathered}
$$




\section{Model based approaches}

Graphical model

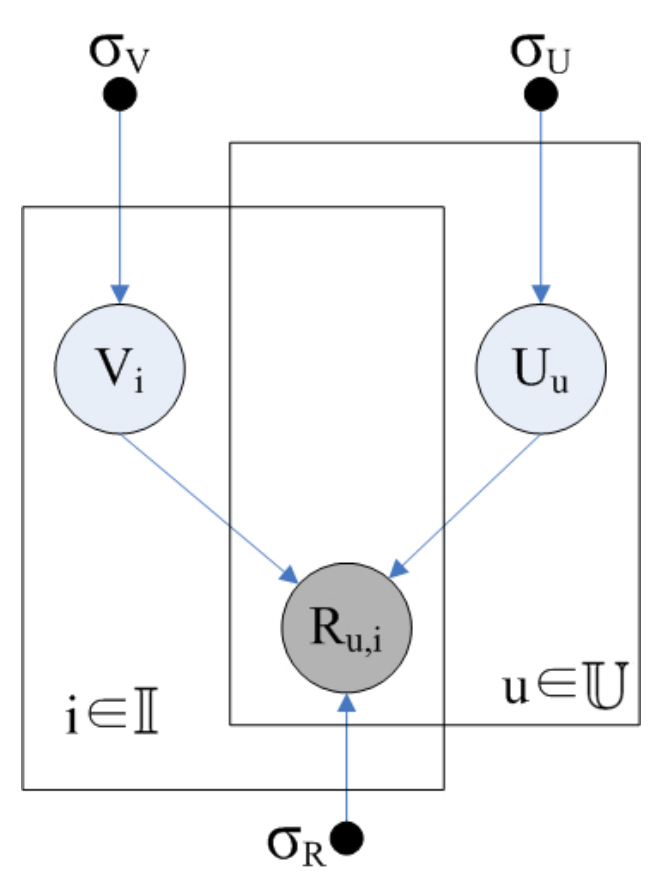

- Learn $U, V$ that minimize

$$
\sum\left(R_{u i}-\widehat{R}_{u i}\right)^{2}+\lambda\left(\|U\|^{2}+\|V\|^{2}\right)
$$

all observed $(u, i)$ 


\section{SoRec}

[Ma et al. 2008]

- Matrix factorization model

- Factorize the ratings and links together.

- Social network as a binary matrix.

- One latent factor for items.

- Two latent factors for users:

- One for the initiator,

- One for the receiver.

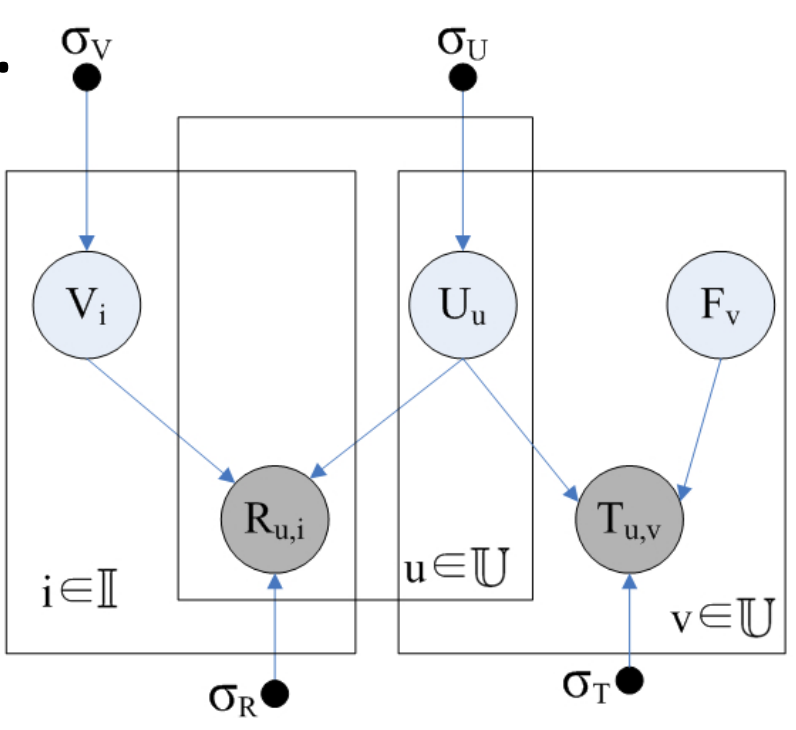

- Same user factor for both contexts (rating actions and social actions). 


\section{FIP}

[Yang et al. 2011]

- Factorizes both rating matrix and the social network.

- Similar to SoRec.

- Assumes undirected network.

- FIP vs. SocRec

- SocRec: Two user factors, FIP one user factor.

- FIP uses user / items features as

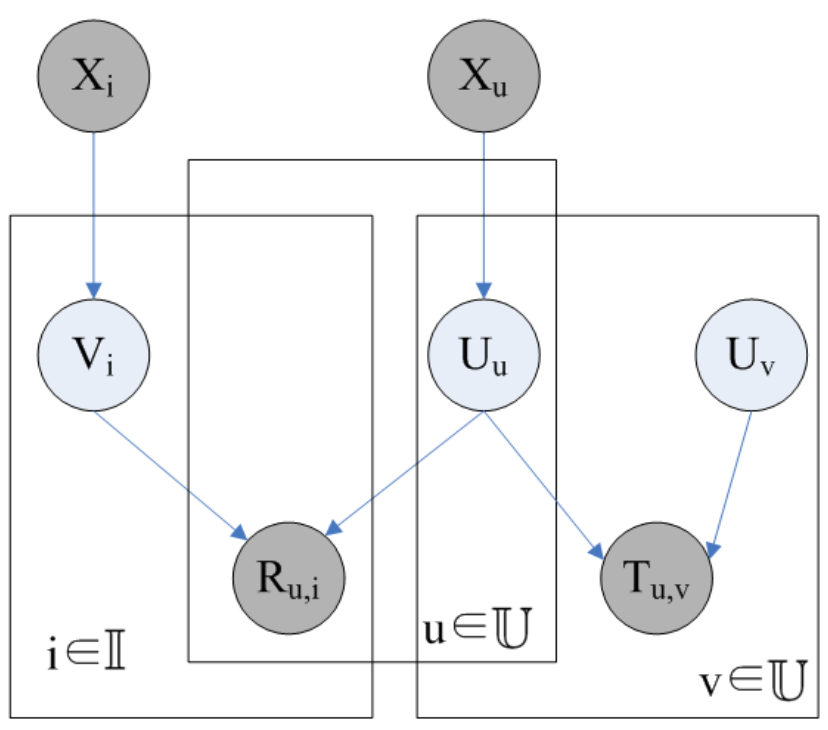
priors for user / item factors. 


\section{Social Trust Ensemble}

[Ma et al. 2009]

- Social Trust Ensemble (STE)

- Linear combination of

- Basic matrix factorization and Latent factors of the user and the item determine the observed rating.

- Social network based approach Latent factors of the neighbors and the latent factor of the item determine the observed rating. 


\section{Social Trust Ensemble}

SFU

- Graphical model

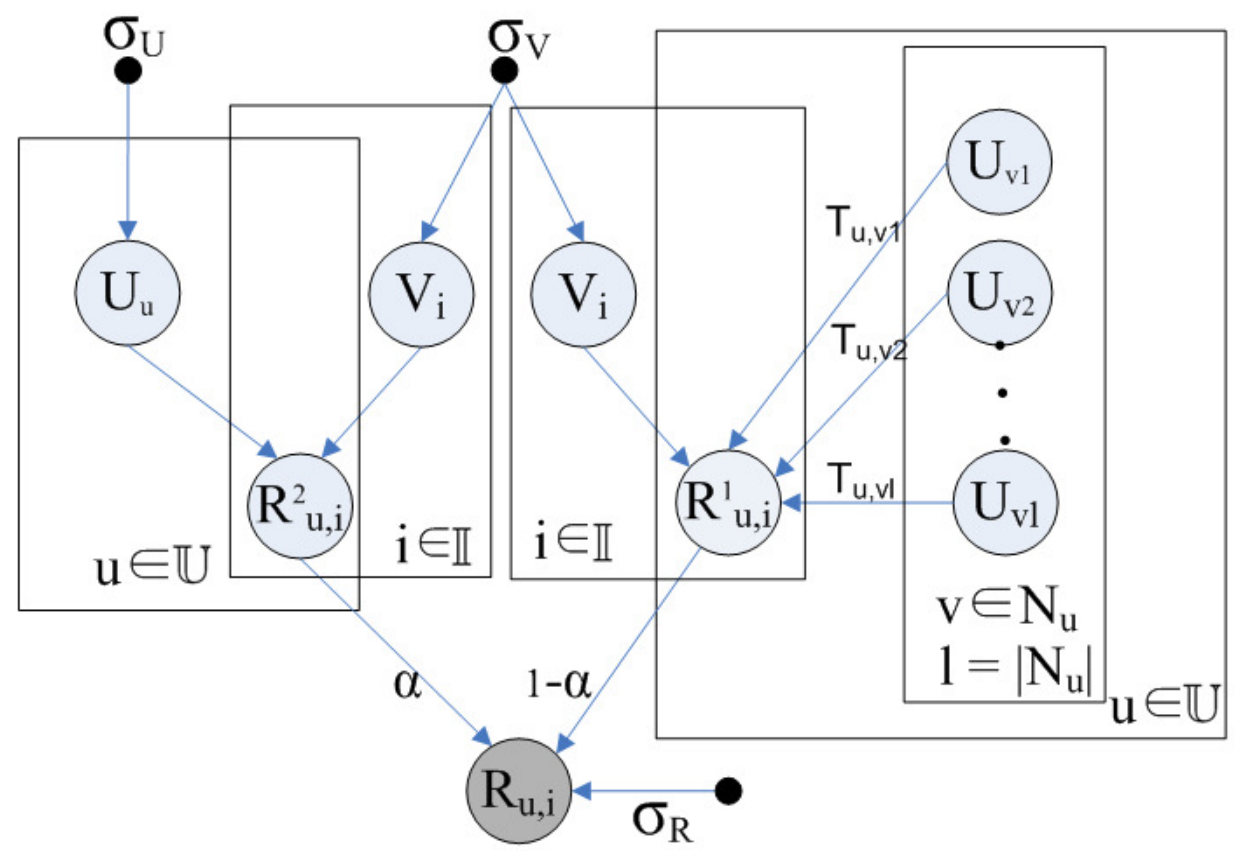

$$
\hat{R}_{u, i}=\alpha U_{u}^{T} V_{i}+(1-\alpha) \sum_{v \in N_{u}} T_{u, v} U_{v}^{T} V_{i}
$$




\section{Social Trust Ensemble}

- Issues with STE

- Learning of user factors is based on observed ratings only.

- STE does not handle trust propagation. 


\section{SocialMF}

[Jamali \& Ester 2010]

- Social influence: behavior of a user $u$ is affected by his direct neighbors $\mathrm{N}_{\mathrm{u}}$.

- Latent factors of a user depend on those of his neighbors.

$$
\widehat{U}_{u}=\sum_{v \in N_{u}} T_{u, v} U_{v}
$$

- $\mathrm{T}_{\mathrm{u}, \mathrm{v}}$ is the normalized trust value. 


\section{SocialMF}

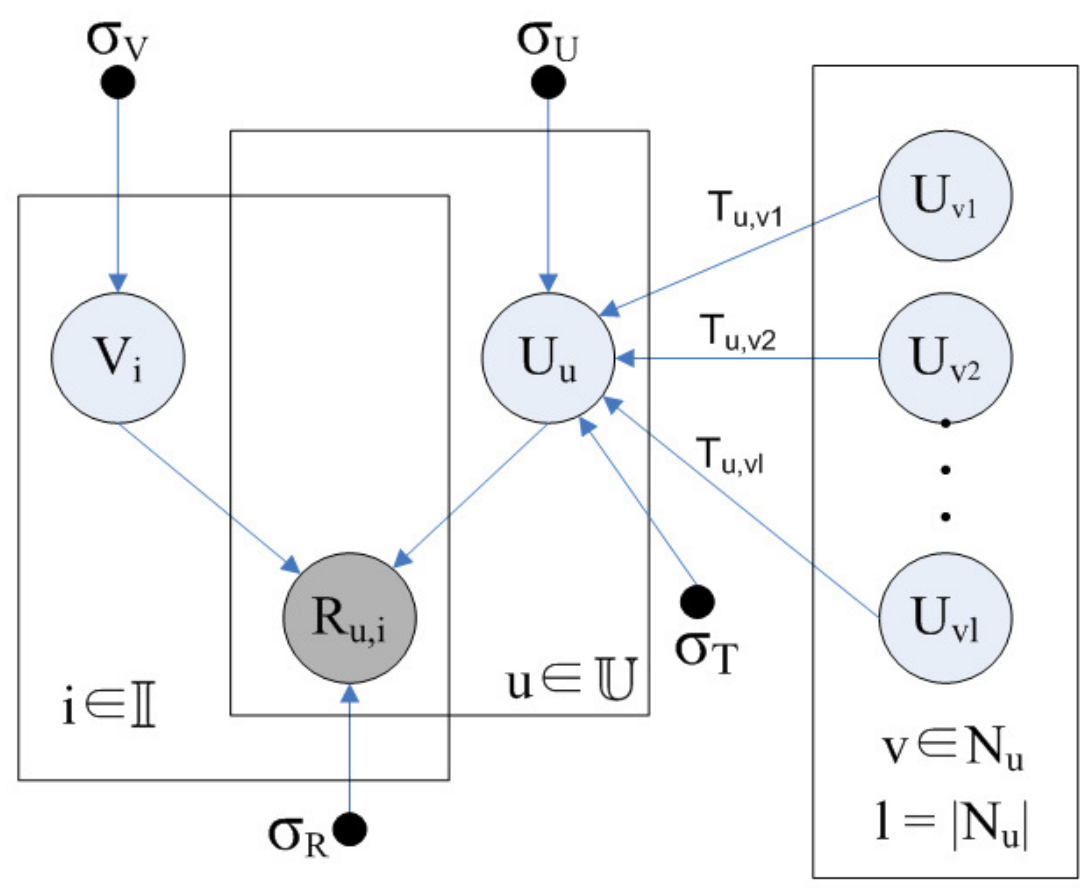

all observed $(u, i)$

$$
\sum\left(R_{u i}-\widehat{R}_{u i}\right)^{2}+\lambda\left(\|U\|^{2}+\|V\|^{2}\right)
$$

$$
+\beta\left(\sum_{u}\left(\left(U_{u}-\sum_{v} T_{u, v} U_{v}\right)\left(U_{u}-\sum_{v} T_{u, v} U_{v}\right)^{T}\right)\right.
$$




\section{SocialMF}

- Properties of SocialMF

- Models trust propagation.

- Learning the user latent factors is possible with social network only.

- Works for cold start users and even users with no ratings.

- Similar ideas in [Ma et al. 2011]. 


\section{Experimental Evaluation}

Results for Epinions

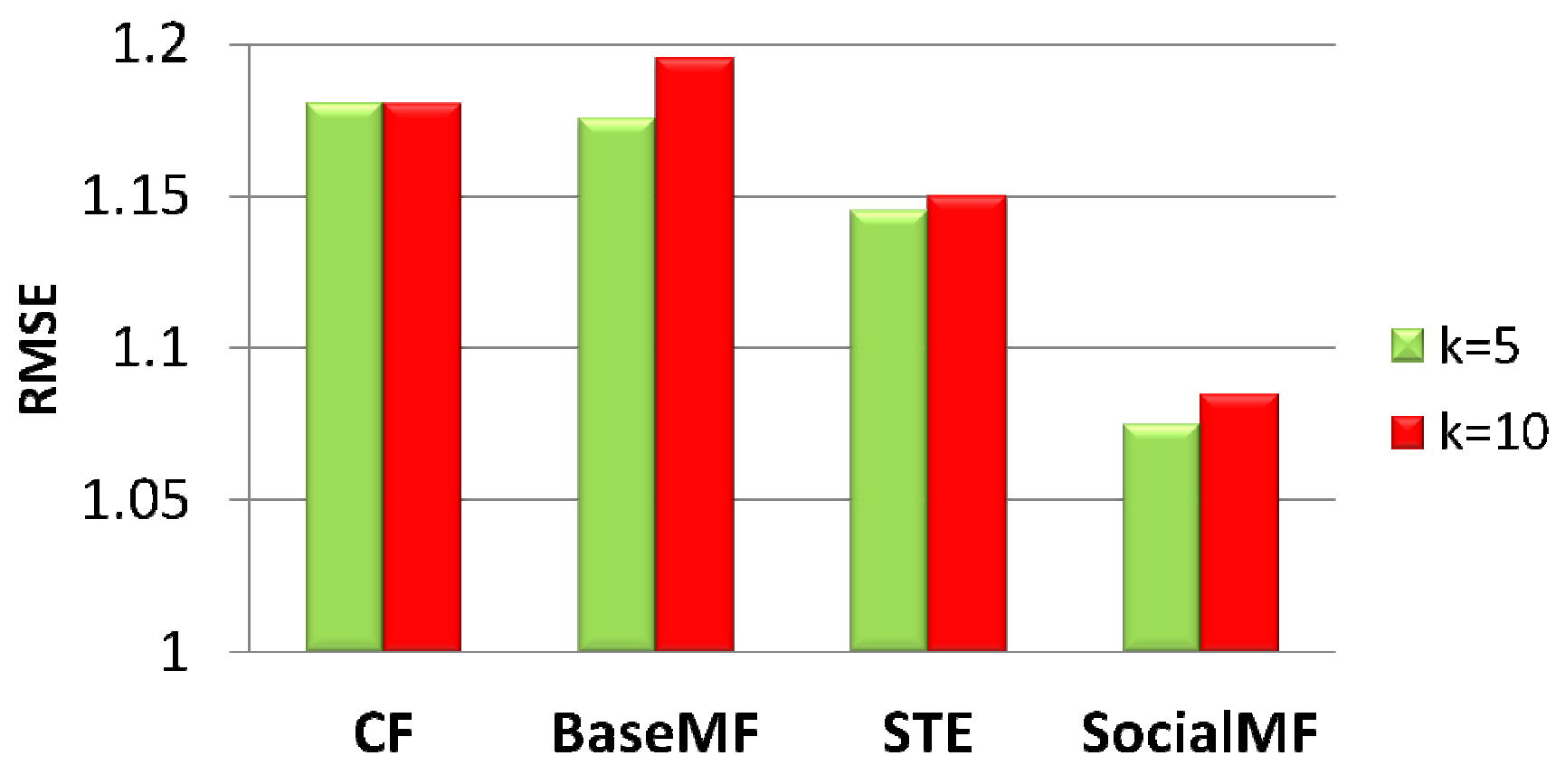

- Gain over STE: $6.2 \%$. for $\mathrm{K}=5$ and $5.7 \%$ for $\mathrm{K}=10$ 


\section{Experimental Evaluation}

Results for Flixster

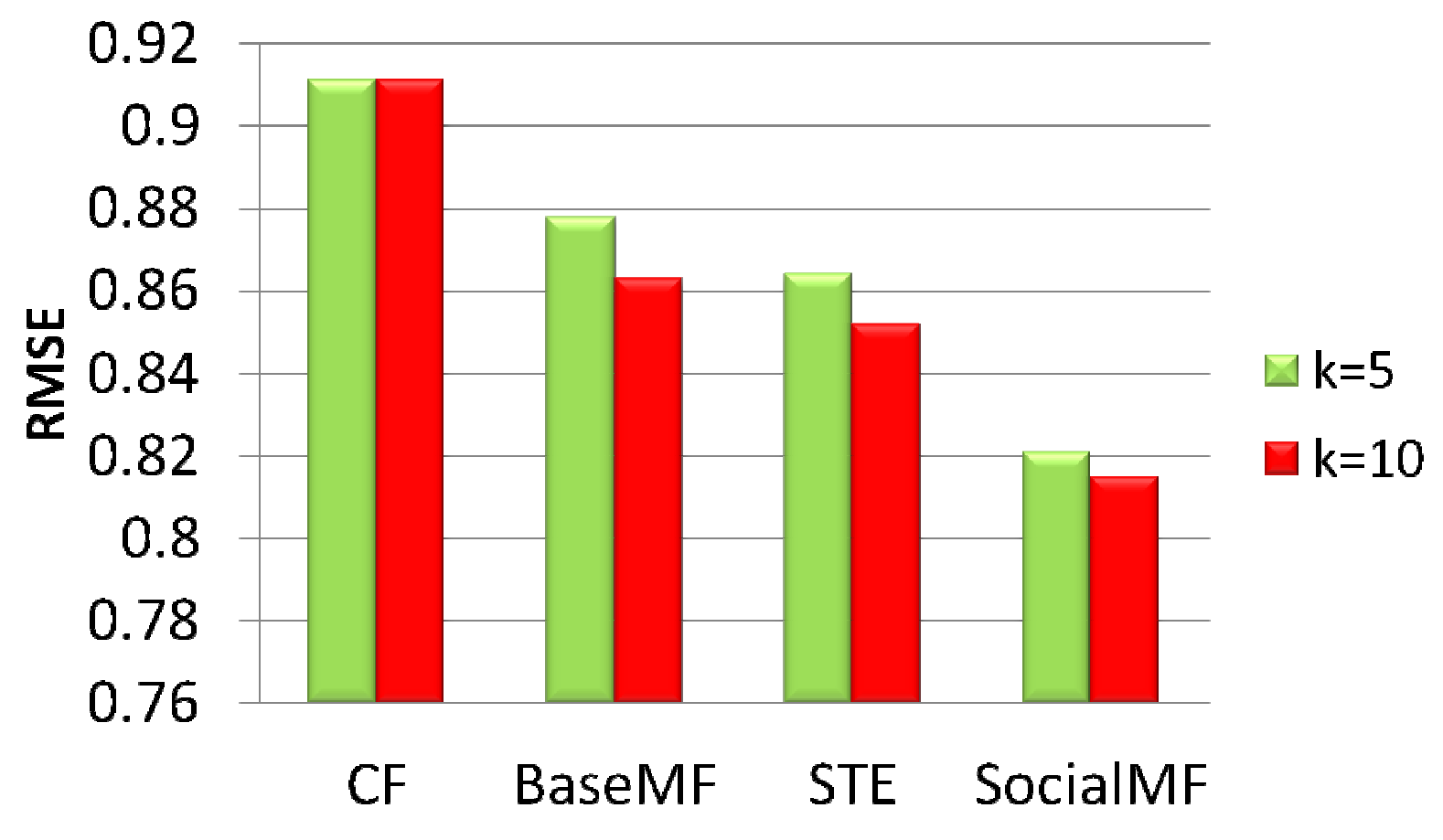

- SocialMF gain over STE (5\%) is 3 times the STE gain over BasicMF (1.5\%) 


\section{Experimental Evaluation}

RMSE Gain of SocialMF over STE

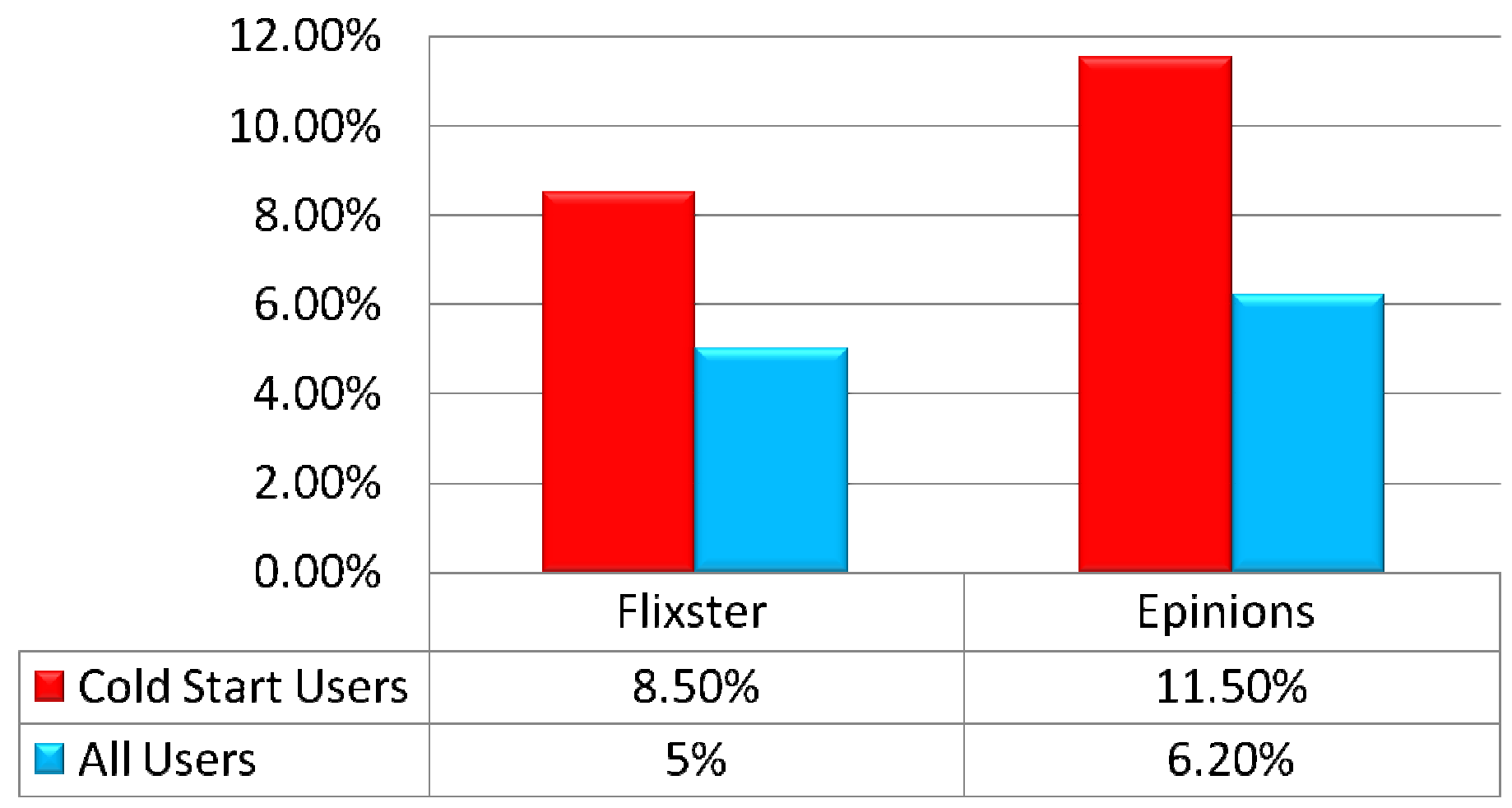




\section{Generalized Stochastic Block Model}

[Jamali et al. 2011]

- Social influence and selection lead to formation of communities/groups.

- Users belong to different (latent) groups, e.g. teacher interacting with students or his/her son or camera being rated by professional vs. amateur.

- Items belong to different (latent) groups, e.g. high-quality and low-quality items.

- Clustering based method for recommendation. 


\section{Generalized Stochastic Block Model}

- Extending mixed membership stochastic block model [Airoldi et al. 2008].

- Users probabilistically act as a member of one of the groups in their actions.

- Every item is considered to belong to a group when it is being rated.

- The relation between users and items is governed by the relation between their groups. 


\section{Generalized Stochastic Block Model}

SFU

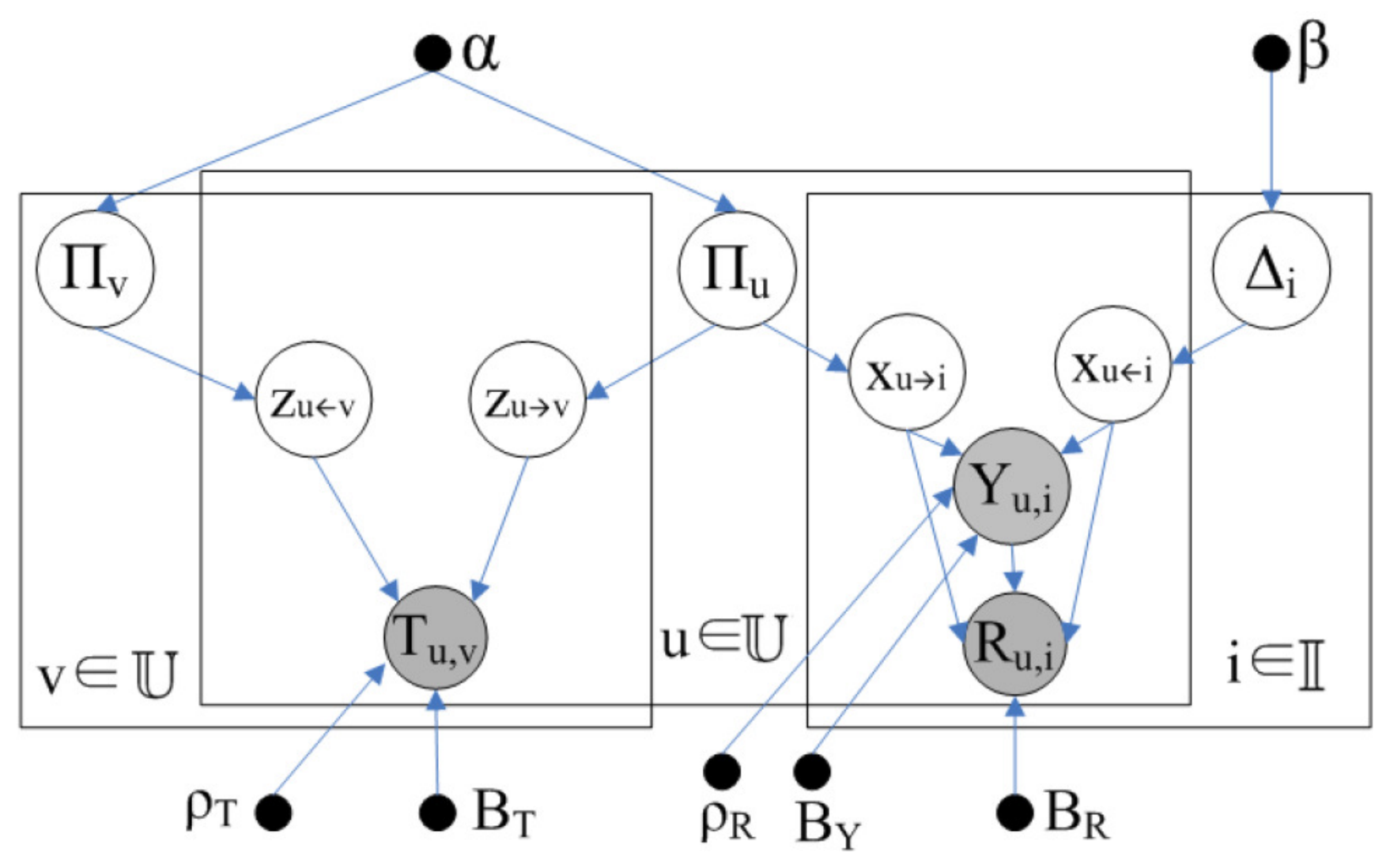




\section{Experimental Evaluation}

SFU

\section{Results for item recommendation}

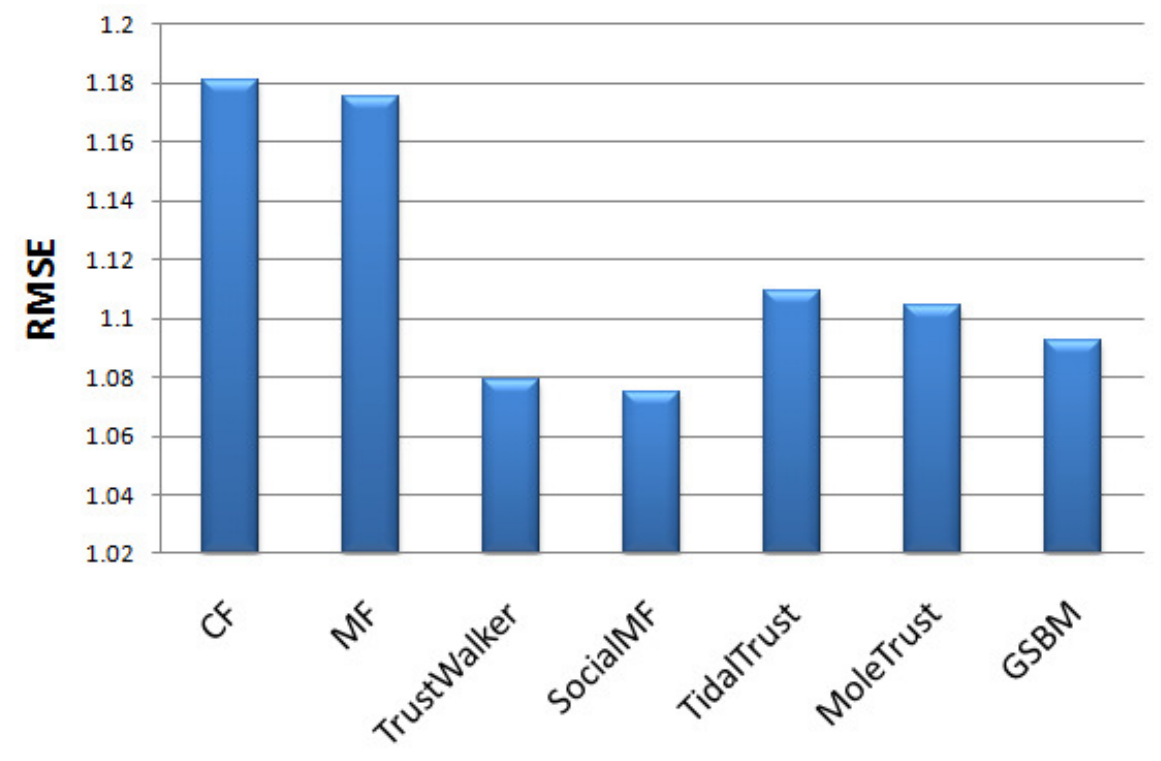

Epinions

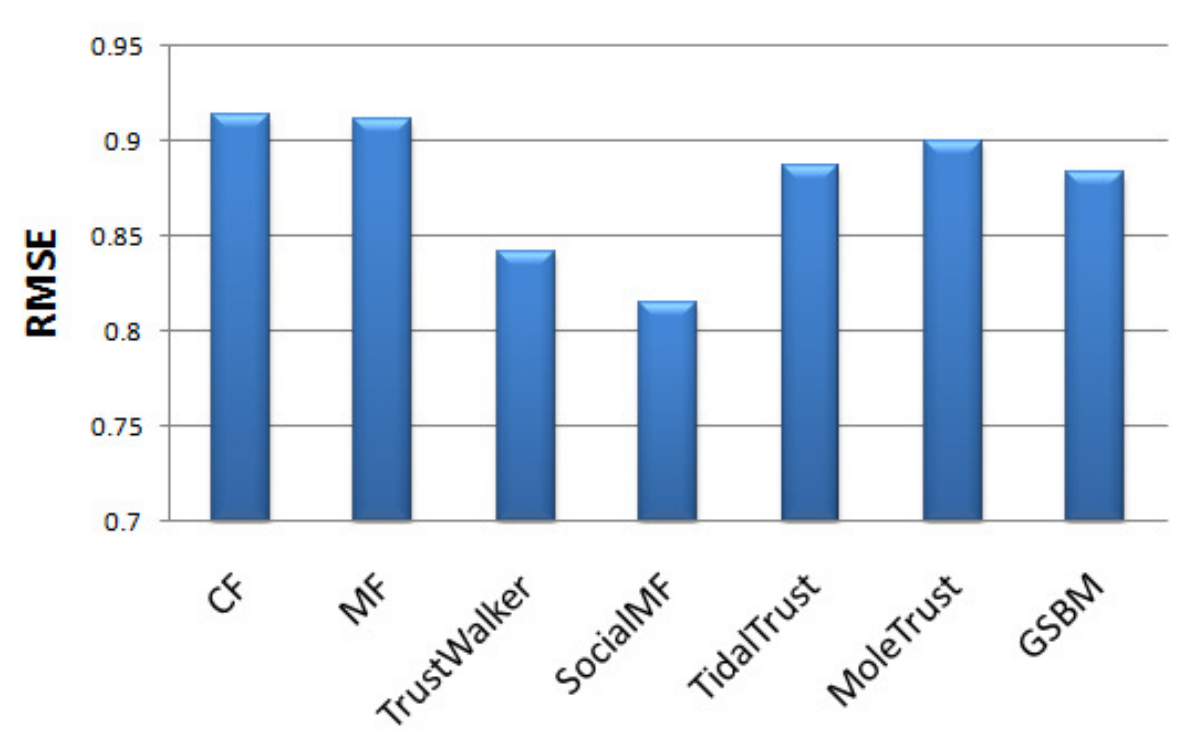

Flixster 


\section{Experimental Evaluation}

\section{Results for friend recommendation}

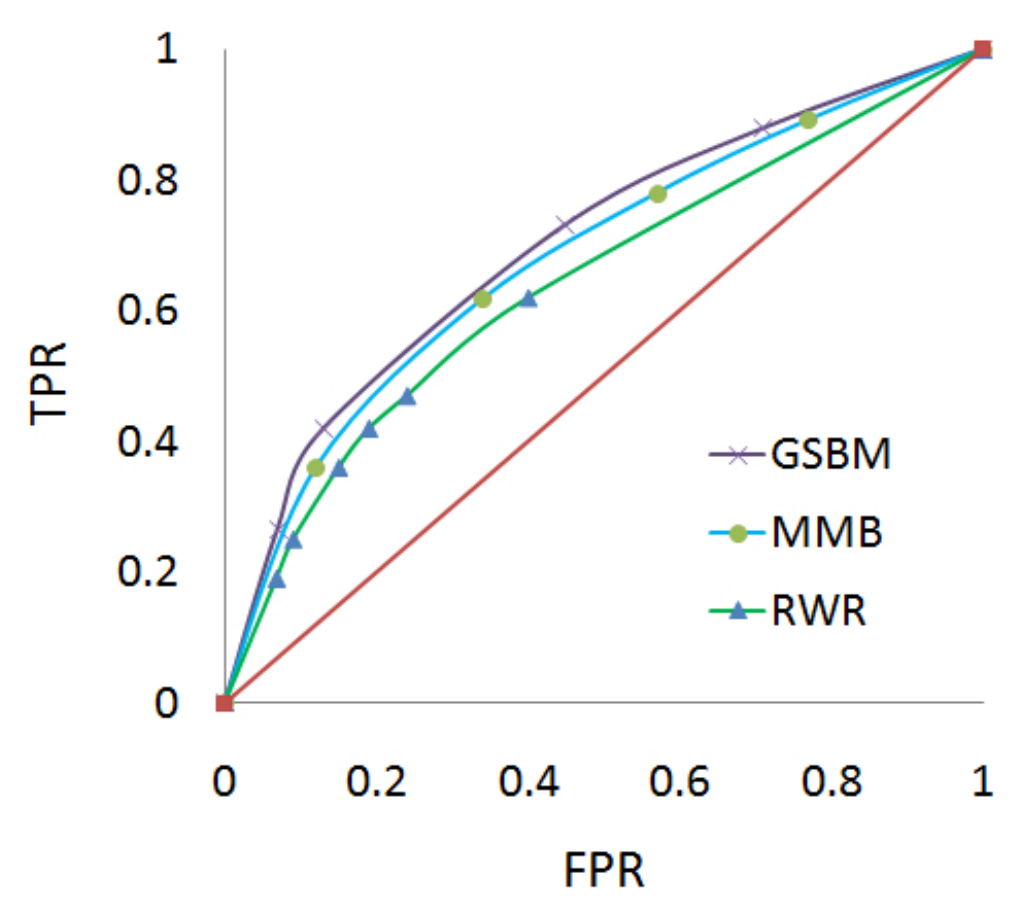

Epinions

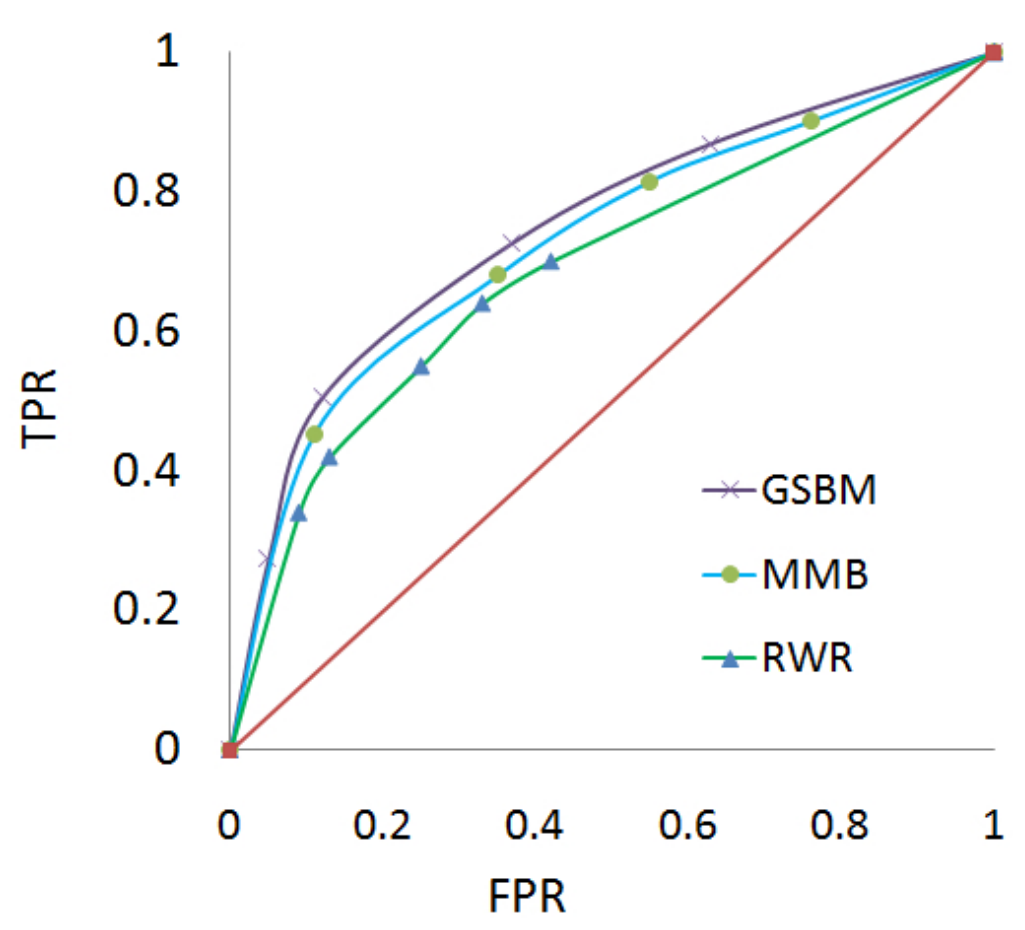

Flixster 


\section{Top-N Item Recommendation}

- So far, we (and most of the literature) have considered only rating prediction.

- Learning objective is to minimize the prediction error computed on the observed ratings in the training set.

- However, top-N recommendation more relevant in practical applications.

- Can use rating prediction method for top-N item recommendation by ranking all items without observed rating in descending order of predicted rating. 


\section{Top-N Item Recommendation}

- Better to change learning objective: minimize the ranking error for all pairs of items whose ratings are observed in the training set.

- Problem 1: This is not efficient.

- Problem 2: Ratings are missing not at random (MNAR). Low ratings are typically much more likely to be missing. [Steck 2010]

- Not a problem for rating prediction, since the data in the training set and the test set are from the same biased distribution. 


\section{Top-N Item Recommendation}

[Yang et al. 2012]

- But a problem for top- $\mathrm{N}$ recommendation, as the $N$ recommended items have to be chosen from all items that were not rated in the training set.

- Solution: compute rating prediction error for all items, not only for those whose ratings are observed in training set.

- Impute small constant rating value $r_{m}$ for unobserved ratings.

- Give smaller weight to prediction errors for unobserved ratings. 


\section{Top-N Item Recommendation}

- Can easily modify existing rating prediction methods for top-N item recommendation.

- E.g. for MF, replace term of the objective function

by

$$
\sum_{\text {all }(u, i)}\left(R_{u i}-\widehat{R}_{u i}\right)^{2}+\lambda\left(\|U\|^{2}+\|V\|^{2}\right)
$$




\section{Experimental Evaluation}

\section{Results (recall top-500) on Epinions}

\begin{tabular}{|l||r|r|r|r|}
\hline \multicolumn{1}{|l||}{$\begin{array}{l}\text { test } \\
\text { users }\end{array}$} & \multicolumn{4}{c|}{ MF models } \\
\cline { 2 - 5 } & No Trust & SocialMF & STE & SoRec \\
\hline \hline \multicolumn{5}{|c|}{ original training (on observed ratings) } \\
\hline all & $1.9 \%$ & $3.5 \%$ & $2.7 \%$ & $2.6 \%$ \\
\hline cold & $1.5 \%$ & $1.0 \%$ & $2.8 \%$ & $2.9 \%$ \\
\hline \hline \multicolumn{5}{|c|}{ modified training (on all ratings) } \\
\hline all & $26.0 \%$ & $29.1 \%$ & $29.4 \%$ & $32.0 \%$ \\
\hline cold & $16.5 \%$ & $27.9 \%$ & $26.6 \%$ & $33.3 \%$ \\
\hline
\end{tabular}

- Modified objective drastically improves recall.

- Relative performance opposite of rating prediction. 


\section{Experimental Evaluation}

\section{Results (recall top-100) on Flixster}

\begin{tabular}{|l||r|r|r|r|}
\hline \multicolumn{1}{|l||}{$\begin{array}{l}\text { test } \\
\text { users }\end{array}$} & \multicolumn{4}{c|}{ MF models } \\
\cline { 2 - 5 } & No Trust & SocialMF & STE & SoRec \\
\hline \hline \multicolumn{5}{|c|}{ original training (on observed ratings) } \\
\hline all & $4.4 \%$ & $4.7 \%$ & $5.3 \%$ & $8.2 \%$ \\
\hline cold & $6.3 \%$ & $6.6 \%$ & $7.2 \%$ & $15.4 \%$ \\
\hline \hline \multicolumn{5}{|c|}{ modified training (on all ratings) } \\
\hline all & $44.3 \%$ & $45.2 \%$ & $47.1 \%$ & $49.1 \%$ \\
\hline cold & $30.8 \%$ & $38.3 \%$ & $47.6 \%$ & $59.2 \%$ \\
\hline
\end{tabular}

- Modified objective drastically improves recall.

- Relative performance opposite of rating prediction. 


\section{Friend Recommendation}

Facebook friend recommendations

- „People you may know“

\section{facebook}

- "Based on mutual friends, work and education information, networks you're part of, contacts and many other factors."

- "Since our formula is automatic, you might occasionally see people you don't know or don't want to be friends with. To remove them from view, just click the X next to their names."

http://www.facebook.com/help/?page $=199421896769556$
Martin Ester: Recommendation in Social Networks, Uutorial at RecSys 2013 


\section{Friend Recommendation}

- Significance of friend recommendation

- All major social networks have it.

\section{facebook Linkedin. $g^{+}$}

- E.g., in Linkedln 50\% of connections from recommendations. [Posse 2012]

- Problem definitions

- Given a user pair $(u, v)$, estimate the probability of creation of the link $u \rightarrow v$.

- Given a user $u$, recommend a list of top users for $u$ to connect to. 


\section{Friend Recommendation}

- How does it work?

- For instance, two people may meet at a party and then get connected on Facebook.

- Reasons to connect often exogeneous to the SN, but the SN contains clues.

- For instance, two people are more likely to meet at a party, if

- They are close to each other in the $\mathrm{SN}$,

- They have similar age,

- They live in the same town. 


\section{Friend Recommendation}

- Friend recommendation vs. item recommendation

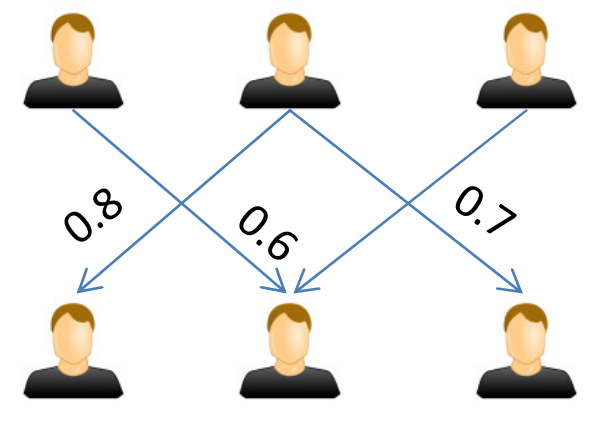

Strength of a relation between

a user and another user

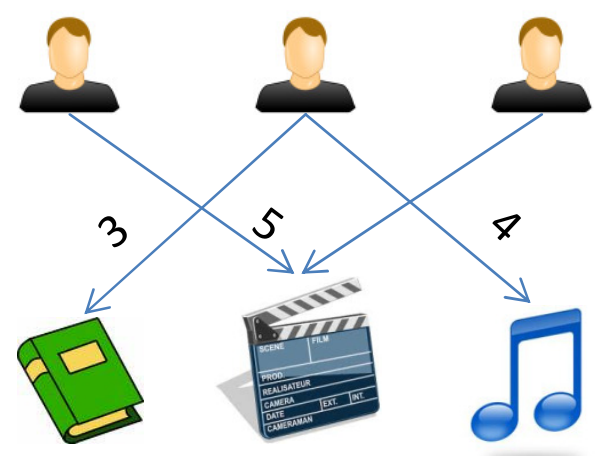

Strength of a relation between

a user and an item 


\section{Friend Recommendation Methods SFU}

- Roots in social selection.

- Users with highest similarity to $u$ are recommended to $u$.

- Every user $u$ is represented by his/her observed properties such as neighbors and past activities such as ratings and clicks. 


\section{Friend Recommendation Methods}

- General similarity measure between users A and B

- the ratio between the amount of information needed to state the commonality of $A$ and $B$ and the information needed to fully describe what $A$ and $B$ are [Lin 1998]:

$$
\operatorname{sim}(A, B)=\frac{\log P(\operatorname{common}(A, B))}{\log P(\operatorname{description}(A, B))}
$$

- Special Cases:

- Cosine similarity

- Pearson correlation

- Jaccard's coefficient 


\section{Topology based Methods}

- Measure similarity, based on direct neighbors of $A$ and $B$.

- Common neighbors $\operatorname{score}(A, B)=\left|N_{A} \cap N_{B}\right|$

- Jaccard's coefficient $\operatorname{score}(A, B)=\frac{\left|N_{A} \cap N_{B}\right|}{\left|N_{A} \cup N_{B}\right|}$

- [Adamic \& Adar 2003]

$$
\operatorname{score}(A, B)=\sum_{C \in N_{A} \cap N_{B}} \frac{1}{\log \left|N_{C}\right|}
$$




\section{Topology based Methods}

- Preferential attachment [Newman 2001]

$$
\operatorname{score}(A, B)=\left|N_{A}\right| \cdot\left|N_{B}\right|
$$

- Initially proposed for modeling network growth.

- SimRank [Jeh \& Widom 2002]

- Two user are similar to the extent that they are joined to similar neighbors.

$$
\begin{aligned}
& \operatorname{score}(A, B)=\gamma \cdot \frac{\sum_{x \in N_{A}} \sum_{y \in N_{B}} \operatorname{score}(x, y)}{\left|N_{A}\right| \cdot\left|N_{B}\right|} \\
& \operatorname{score}(x, x)=1
\end{aligned}
$$




\section{Path based Methods}

- Measure similarity, based on paths between A and $\mathrm{B}$.

- Katz [Katz 1953]:

$$
\operatorname{score}(A, B)=\sum_{l=1}^{\infty}\left(\beta^{l} \cdot \operatorname{paths}_{A, B}^{l}\right)
$$

- path ${ }_{\mathrm{A}, \mathrm{B}}$ : number of paths of length $l$ from $A$ to $B$

- Hitting time [Liben-Nowell \&Kleinberg 2003]

- score $(A, B)$ : Average number of steps for a random walk from $A$ to $B$. 


\section{Path based Methods}

- Random walk with restart [Pan et al. 2004]

- A random walk starts from A. At each step, with probability $\alpha$ the random walk restarts.

$-\operatorname{score}(A, B)$ :

probability of being at $B$ during random walk from $A$. 


\section{Path based Methods}

- Supervised random walks [Backstrom \& Leskovec 2011]

- Learn edge weights so that random walk visits more likely "positive" nodes (to which new edges will be created in the future) than "negative" nodes (other nodes).

- Supervised learning task: given a source node $s$ and positive and negative training examples, learn a function that assigns edge weights (i.e., random walk transition probabilities) so that a weighted random walk has a higher probability of visiting positive examples than negative examples. 


\section{Path based Methods}

- Each edge $(u, v)$ has a corresponding feature vector $\varphi_{u v}$ that describes

- The nodes $u$ and $v$ (e.g., age, gender, hometown), and

- The interaction attributes (e.g., when the edge was created, how many messages $u$ and $v$ exchanged, or how many photos they appeared together in).

- Function $f_{w}$ takes the edge feature vector $\varphi_{u v}$ as input and computes the corresponding edge weight $a_{u v}$.

- Goal is to learn parameter $w$ of function $f_{w}$. 


\section{Path based Methods}

- Objective function

$$
\min _{w} F(w)=\|w\|^{2}+\lambda \sum_{d \in D, l \in L} h\left(p_{l}-p_{d}\right)
$$

- $D$ is the set of positive nodes, $L$ the set of negative nodes,

$-P_{i}$ is the probability of visiting node $i$, and

$-h$ is a loss function with

$$
h(.)=0 \text { for } p_{d}>p_{l} \text { and } h(.)>0 \text { for } p_{d}<p_{l} \text {. }
$$




\section{Path based Methods}

- Experimental results on Co-authorship dataset

\begin{tabular}{l||r|r} 
Learning Method & AUC & Prec@20 \\
\hline \hline Random Walk with Restart & 0.63831 & 3.41 \\
Adamic-Adar & 0.60570 & 3.13 \\
Common Friends & 0.59370 & 3.11 \\
Degree & 0.56522 & 3.05 \\
\hline DT: Node features & 0.60961 & 3.54 \\
DT: Network features & 0.59302 & 3.69 \\
DT: Node+Network & 0.63711 & 3.95 \\
DT: Path features & 0.56213 & 1.72 \\
DT: All features & 0.61820 & 3.77 \\
\hline LR: Node features & 0.64754 & 3.19 \\
LR: Network features & 0.58732 & 3.27 \\
LR: Node+Network & 0.64644 & 3.81 \\
LR: Path features & 0.67237 & 2.78 \\
LR: All features & 0.67426 & 3.82 \\
\hline SRW: one edge type & 0.69996 & 4.24 \\
SRW: multiple edge types & $\mathbf{0 . 7 1 2 3 8}$ & $\mathbf{4 . 2 5}$
\end{tabular}

-DT: decision tree

-LR: logistic regression

-SRW: supervised rand.walk

- Most methods have similar performance

- LR is strongest competitor

- SRW outperforms LR 


\section{Path based Methods}

- Experimental results on Facebook dataset

\begin{tabular}{l||r|r} 
Learning Method & AUC & Prec@20 \\
\hline \hline Random Walk with Restart & 0.81725 & 6.80 \\
Adamic-Adar & 0.81586 & 7.35 \\
Common Friends & 0.80054 & 7.35 \\
Degree & 0.58535 & 3.25 \\
\hline DT: Node features & 0.59248 & 2.38 \\
DT: Network features & 0.76979 & 5.38 \\
DT: Node+Network & 0.76217 & 5.86 \\
DT: Path features & 0.62836 & 2.46 \\
DT: All features & 0.72986 & 5.34 \\
\hline LR: Node features & 0.54134 & 1.38 \\
LR: Network features & 0.80560 & 7.56 \\
LR: Node+Network & 0.80280 & 7.56 \\
LR: Path features & 0.51418 & 0.74 \\
LR: All features & 0.81681 & 7.52 \\
\hline SRW: one edge type & $\mathbf{0 . 8 2 5 0 2}$ & 6.87 \\
SRW: multiple edge types & $\mathbf{0 . 8 2 7 9 9}$ & 7.57
\end{tabular}

-DT: decision tree

-LR: logistic regression

-SRW: supervised rand.walk

-Unsupervised methods and LR perform very well

- SRW performs best 


\section{Model based Methods}

- MF based models [Rennie \& Srebo 2005]

- Social network as a binary matrix.

- Similar to MF methods for rating prediction.

- Factorize the network matrix into product of lower rank matrices (representing user factors).

- Advanced version in [Yang et al. 2011]: factorize user-user matrix and user-item matrix simultaneously. 


\section{Future Research Directions}

SFU

- Recommendation in heterogeneous networks with more than 2 entity types

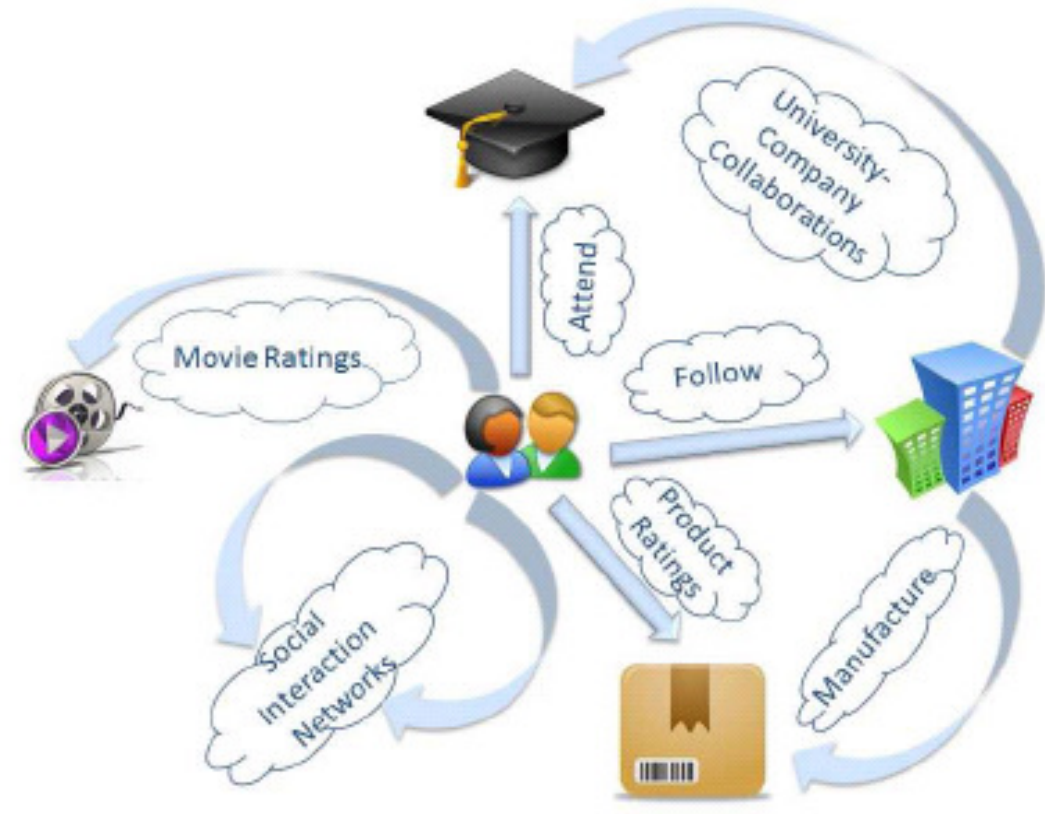

- How to recommend entities of one type to entities of another type?

Martin Ester: Recommendation in Social Networks, Tutorial at RecSys 2013 


\section{Future Research Directions}

- Collective Matrix Factorization [Singh \& Gordon 2008]

- Simultaneously factorize the matrices of all binary relationships.

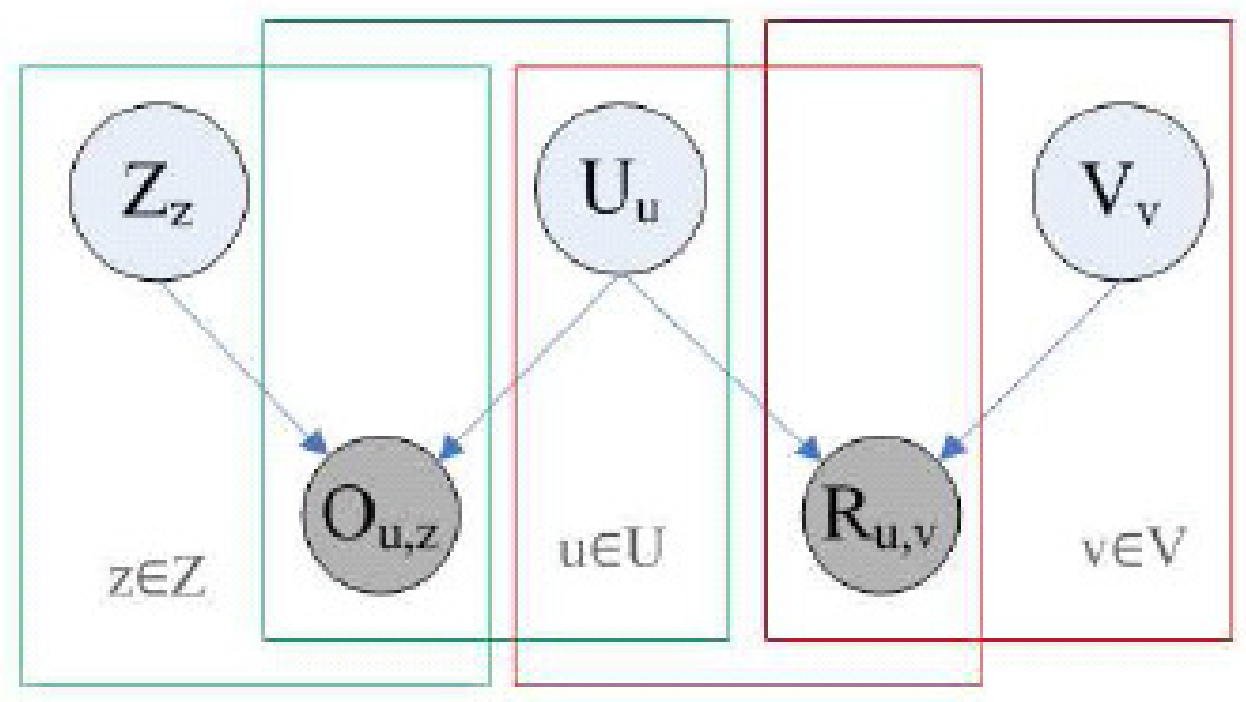

- An entity uses the same latent factors in all of its relationships (contexts). 


\section{Future Research Directions}

- HeteroMF: Context Dependant Factor Models [Jamali \& Lakshmanan 2013]

- Each entity has a base latent factor.

- For each relationship (context) in which its entity type participates, the entity has context specific latent factors.

- Context specific factors are derived from the base factors employing a transfer matrix. 


\section{Future Research Directions}

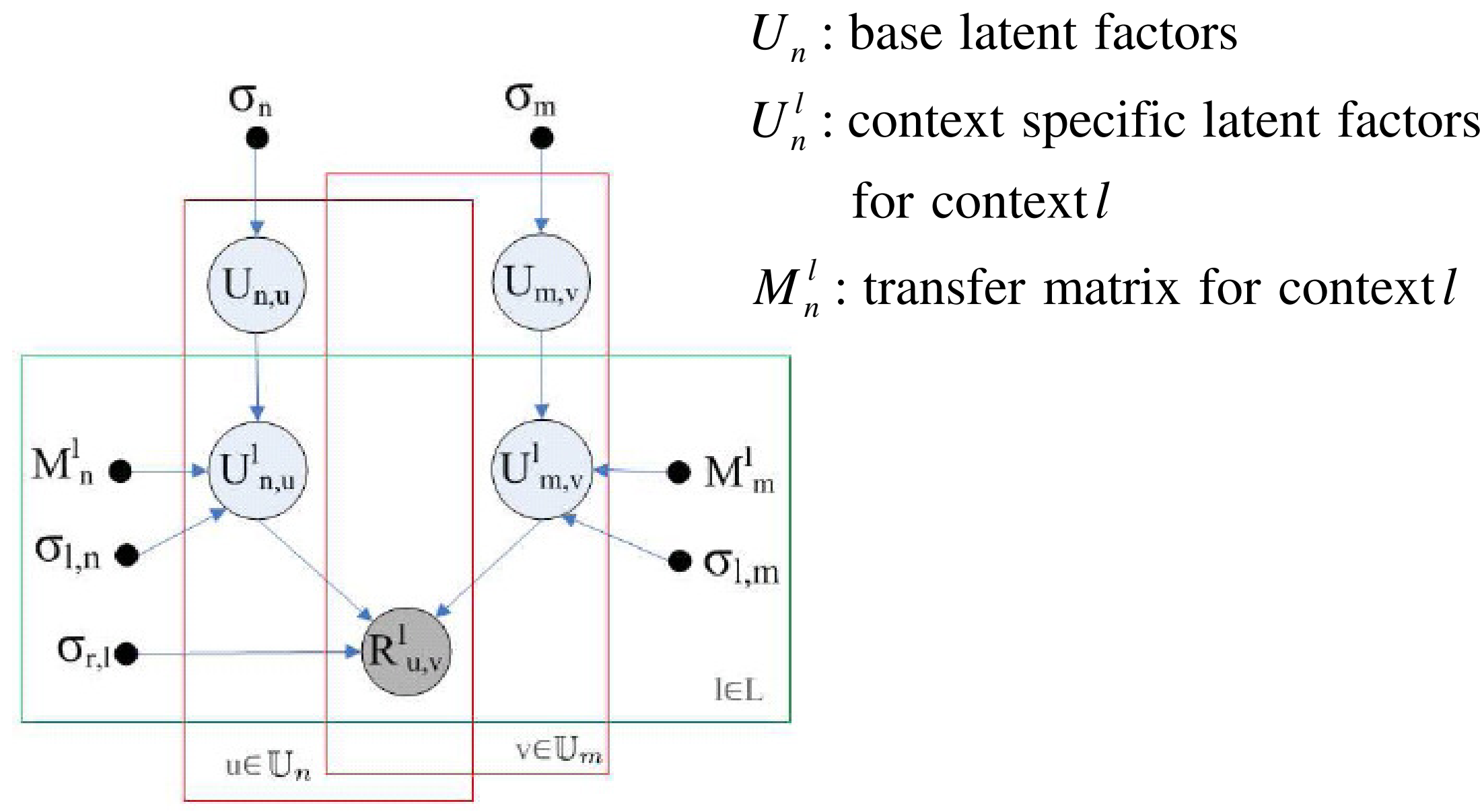




\section{Future Research Directions}

- Explanation of social recommendations

- [Papadimitriou et al. 2012] distinguishes the following types of explanations (and their hybrids).

- Human style of explanation, based on similar users.

- Item style of explanation, based on choices made by the user on similar items.

- Feature style of explanation, based on features of items that were previously rated by the user. 


\section{Future Research Directions}

- Do social explanations work? [Sharma \& Cosley 2013]

- Distinguish the following types of explanations:

- Overall Popularity: The number of Likes by all Facebook users for an artist.

- Friend Popularity: The number of friends of a user who Like an artist.

- Random Friend: The name of a random friend, chosen from those that Like an artist.

- Good Friend: The name of a close friend, chosen from those that Like an artist.

- Good Friend \& Count: A combination of Good Friend and

MartiFFiendmPopularity. Networks, Tutorial at RecSys 2013 


\section{Future Research Directions}

- Persuasiveness of explanations

- For each recommendation, we ask the user how likely (on scale [0..10]) is she to check out the recommended artist.

\begin{tabular}{lrcc}
\hline Explanation Strategy & N & Mean & Std. Dev. \\
\hline FriendPop & 1203 & 2.12 & 2.42 \\
RandFriend & 1225 & 2.08 & 2.49 \\
OverallPop & 1191 & 2.36 & 2.69 \\
GoodFriend & 434 & 2.52 & 2.69 \\
GoodFrCount & 405 & 2.71 & 2.90 \\
\hline
\end{tabular}

- Showing the right friend matters.

- Popularity only matters if user identifies with the crowd. 


\section{Future Research Directions}

- Informativeness of explanations

- How effective are explanations in directing users to items that receive high consumption ratings?

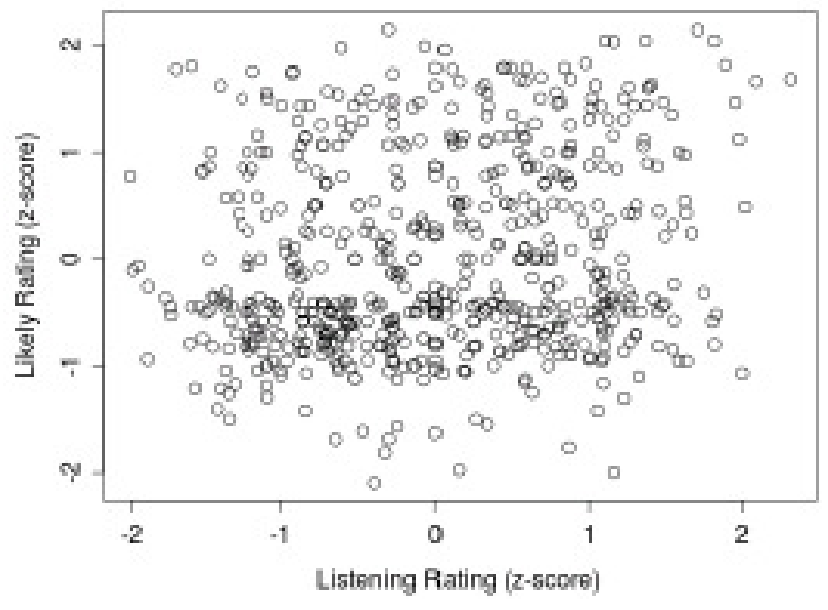

- The persuasiveness and informativeness of an explanation are quite different. 


\section{Future Research Directions}

- Privacy of recommendation in social networks

- How to preserve privacy when employing social networks?

- Recommendation in mobile social networks

- Can we develop distributed algorithms?

- How to exploit the user location?

- Recommendation in social networks with user-generated content

- How can we integrate topic models? 


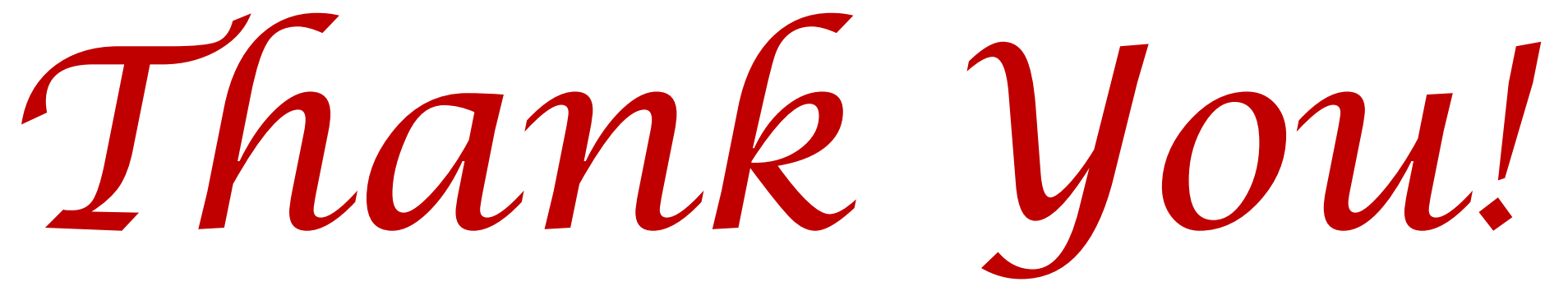




\section{References}

- $\quad$ L. A. Adamic, Eytan Adar. Friends and neighbors on the web. Social Networks, 25(3):211-230, July 2003.

- $\quad$ E. M. Airoldi, D. M. Blei, S. E. Fienberg, E. P. Xing. Mixed membership stochastic blockmodels. Journal of Machine Learning Research, 9:1981-2014, 2008.

- A. Anagnostopoulus, Kumar R., Mahdian M.: Influence and correlation in social networks, KDD 2008.

- L. Backstrom, J. Leskovec. Supervised Random Walks: Predicting and Recommending Links in Social Networks, WSDM 2011.

- J. Golbeck. Computing and Applying Trust in Web-based Social Networks. PhD thesis, University of Maryland College Park, 2005.

- M. Gomez-Rodriguez, J. Leskovec, A. Krause: Inferring networks of diffusion and influence, KDD 2010.

- M. Jamali, M. Ester: A Matrix Factorization Technique with Trust Propagation for Recommendation in Social Networks, Recsys 2010.

- M. Jamali, M. Ester: TrustWalker : A Random Walk Model for Combining Trust-based and Item-based Recommendation, KDD 2009.

- M. Jamali, T. Huang, M. Ester: A Generalized Stochastic Block Model for Recommendation in Social Rating Networks, ACM RecSys 2011.

- $\quad$ M. Jamali, L. Lakshmanan: HeteroMF: Recommendation in Heterogeneous Information Networks using Context Dependent Factor Models, WWW 2013. 


\section{References}

- $\quad$ G. Jeh, J. Widom: Simrank: a measure of structural-context similarity, KDD 2002.

- L. Katz: A new status index derived from sociometric analysis, Psychometrika, 18(1):39-43, 1953.

- $\quad$ Y. Koren, R. Bell R., C. Volinsky: Matrix factorization techniques for recommender systems, IEEE Computer, 2009.

- D. Liben-Nowell, J. Kleinberg. The Link Prediction Problem for Social Networks, CIKM 2003.

- D. Lin: An information-theoretic definition of similarity, ICML 1998.

- H. Ma, I. King, M. R. Lyu: Learning to recommend with social trustensemble, SIGIR 2009.

- H. Ma, M. R. Lyu, Irwin King: Learning to recommend with trust and distrust relationships, RecSys 2009.

- H. Ma, H. Yang, M. R. Lyu, I. King. Sorec: social recommendation using probabilistic matrix factorization, CIKM 2008.

- $\quad$ P. Massa, P. Avesani. Trust-aware recommender systems, RecSys 2007.

- $\quad$ S.A. Myers, J. Leskovec: On the convexity of latent social network inference, NIPS 2010.

- P.R. Monge, N.S. Contractor: Theories of communication networks, Oxford University Press, 2003.

- $\quad$ M. E. J. Newman. Clustering and preferential attachment in growing networks, 2001.

- J.-Y. Pan, H.-J. Yang, C. Faloutsos, P. Duygulu. Automatic multimedia cross-modal correlation discovery, KDD 2004. 


\section{References}

- A. Papadimitriou, P. Symeonidis, Y. Manolopoulos: A generalized taxonomy of explanations styles for traditional and social recommender systems, Data Mining and Knowledge Discovery, 24(3), 555583, 2012.

- C. Posse: Key Lessons Learned Building Recommender Systems for Large-Scale Social Networks, KDD 2012.

- J.D.M. Rennie, N. Srebro. Fast maximum margin matrix factorization for collaborative prediction, ICML 2005.

- A. P. Singh, G. J. Gordon. Relational learning via collective matrix factorization, KDD 2008.

- A. Sharma, D. Cosley: Do social explanations work? Studying and modeling the effects of social explanations in recommender systems, WWW 2013.

- H. Steck: Training and testing of recommender systems on data missing not at random, KDD 2010.

- S. Wasserman, K. Faust: Social network analysis: methods and applications, Cambridge University Press, 1994 .

- S.H. Yang, B. Long, A. Smola, N. Sadagopan, Z. Zheng, H. Zha: Like like alike - Joint Friendship and Interest Propagation in Social Networks, WWW 2011.

- $\quad$ X. Yang, H.Steck, Y. Guo, Y. Liu: On top-k recommendation using social networks, RecSys 2012. 